\title{
Non-intrusive stochastic analysis with parameterized imprecise probability models: II. reliability and rare events analysis
}

\author{
Pengfei Wei ${ }^{a^{*}, c}$, Jingwen Song ${ }^{b *, c}$, Sifeng $\mathrm{Bi}^{c}$, Matteo Broggi ${ }^{c}$, Michael Beer ${ }^{c, d, e}$, Zhenzhou $\mathrm{Lu}^{b}$, \\ Zhufeng Yue ${ }^{a}$ \\ ${ }^{a}$ School of Mechanics, Civil Engineering and Architecture, Northwestern Polytechnical \\ University, Xi'an 710072, China \\ ${ }^{b}$ School of Aeronautics, Northwestern Polytechnical University, Xi'an 710072, China \\ ${ }^{c}$ Institute for Risk and Reliability, Leibniz Universität Hannover, Callinstr. 34, \\ Hannover, Germany \\ ${ }^{d}$ Institute for Risk and Uncertainty, University of Liverpool, Peach Street, L69 7ZF \\ Liverpool, United Kingdom \\ ${ }^{e}$ International Joint Research Center for Engineering Reliability and Stochastic \\ Mechanics, Tongji University, Shanghai 200092, China
}

\begin{abstract}
Structural reliability analysis for rare failure events in the presence of hybrid uncertainties is a challenging task drawing increasing attentions in both academic and engineering fields. Based on the new imprecise stochastic simulation framework developed in the companion paper, this work aims at developing efficient methods to estimate the failure probability functions subjected to rare failure events with the hybrid uncertainties being characterized by imprecise probability models. The imprecise stochastic simulation methods are firstly improved by the active learning procedure so as to reduce the computational costs. For the more challenging rare failure events, two extended subset simulation based sampling methods are proposed to provide better performances in both local and global parameter spaces. The computational costs of both methods are the same with the classical subset simulation method. These two methods are also combined with the active learning procedure so as to further substantially reduce the computational costs. The estimation errors of all the methods are analyzed based on sensitivity indices and statistical properties of the developed estimators. All these new developments enrich the imprecise stochastic simulation framework. The feasibility and efficiency of the proposed methods are demonstrated with numerical and engineering test examples.
\end{abstract}

Keywords: Aleatory uncertainty; Epistemic uncertainty; Imprecise probability; Subset simulation; Highdimensional model representation; Imprecise stochastic simulation; Uncertainty quantification; Failure probability; Sensitivity analysis.

\section{Introduction}

In structural engineering, uncertainties are ubiquitous among the parameters of computational models due to their intrinsic random property, lack of information, etc. Characterizing these uncertainties and propagating them through the computational models so as to learn the risk and reliability have been two main tasks in the deign process of the structures. As it has been introduced in the companion paper ${ }^{[1]}$,

\footnotetext{
${ }^{*}$ Corresponding author:

pengfeiwei@nwpu.edu.cn (P. Wei) and jwsong0879@163.com (J. Song)

Please cite this paper as: Wei, P., Song, J., Bi, S., Broggi, M., Beer, M., Lu, Z., \& Yue, Z. (2019). Non-intrusive stochastic analysis with parameterized imprecise probability models: II. Reliability and rare events analysis. Mechanical Systems and Signal Processing, https://doi.org/10.1016/j.ymssp.2019.02.015
} 
uncertainty characterization models can be generally divided into three groups, i.e., the precise probability model, non-probabilistic models and imprecise probability models, where the last category can be seen as combination of the previous two, and has been demonstrated to be the most appealing approach for characterizing aleatory and epistemic uncertainties separately in a unified framework, especially in the design process of structures where the available information may be incomplete and/or imprecise ${ }^{[2]}$. Commonly used imprecise probability models include evidence theory ${ }^{[3]}$, probability-box (p-box) ${ }^{[4]}$, fuzzy probability model ${ }^{[5]}$, second-order probability model ${ }^{[6]}$, etc., where those parameterized ones such as parameterized p-box have attracted the most attentions due to the simplicity and ease of applications ${ }^{[7]}$. Thus, propagating the parameterized imprecise probability models through the expensive computational models has drawn more and more attentions in recent years. In the companion paper, we have comprehensively studied the estimation of the probabilistic response functions by developing two sampling techniques, and in this paper, we focus on the estimation of the failure probability functions with emphasis on rare failure events.

For precise probability models, the purpose of uncertainty propagation is to estimate the failure probability, and in the past decades, tremendous methods have been developed for this purpose. These methods can generally be divided into three categories, i.e., the approximate analytical methods (e.g., the FORM and SORM methods) ${ }^{[8][9]}$, the sampling methods (e.g., the importance sampling and the subset simulation (SS) methods) ${ }^{[10]-[12]}$ and the adaptive surrogate model methods (e.g., the AK-MCS method) [13][14]. The adaptive surrogate model methods, combining the sampling techniques, active learning procedure, and surrogate models, have received the most attentions due to their wide applicability and high efficiency ${ }^{[15][16]}$. The reliability analysis problems in the context of precise probability models have been comprehensively studied, however, when it comes to imprecise probability models, the current research is relatively limited in the literatures.

The non-probabilistic models, due to its simplicity, have also been widely utilized for uncertainty quantification. For example, in Ref. [17], the inverse uncertainty quantification problem was studied by using the multivariate interval models under scarce data, and compared with the one based on classical Bayesian updating; in Ref. [18], the correlation estimation has been considered for multivariate interval model; in Ref. [19], the structural reliability analysis subjected to multivariate interval inputs was treated based on an enhanced subinterval method. Commonly, in the propagation of non-probabilistic models, an optimization procedure directly performed on the model response function is inevitable.

When the imprecise probability models are concerned, the estimations of failure probability bounds ${ }^{[20]}$ or failure probability functions ${ }^{[21][22]}$ are commonly of interest, and many classical methods developed for precise probability model have been extended to deal with this type of problems. The available methods can also be divided into three categories, i.e., the approximate analytical methods, the surrogate model methods and the sampling methods. In Ref. [23], the FORM and SORM methods have been extended for estimating the failure probability bounds in the context of evidence theory by introducing the concept of "most-probable focal element". The moment methods have also been extended for estimating the failure probability bounds in the context of parameterized p-box model by estimating the response moment bounds and approximating the response probability density function (PDF) with a prespecified distribution type ${ }^{[24]}$. The estimation errors of this type of methods come from the approximation 
errors of the response functions as well as the errors between the assumed and the true distribution types of the responses, both of which are difficult to assess and handle.

The surrogate model methods for estimating the failure probability bounds are also implemented in an active learning manner. In Ref. [25], the classical AK-MCS method is extended for estimating the failure probability bounds in the context of evidence theory by approximating the signs of the maximum and minimum values of the response functions with the Kriging surrogate model and active learning procedure. In Ref. [26], a two-level Kriging surrogate model combined with the active learning procedure is proposed to cope with the estimation of the failure probability bounds with the input variables being characterized by either parametric or non-parametric p-box models. The estimation errors of these methods mainly come from the approximation errors of the surrogate models, which are easy to control due to the excellent statistical properties of the Kriging surrogate models.

The sampling methods have also been developed for reliability analysis in the context of imprecise probability models, among which two proprietary methods, i.e., Extended Monte Carlo simulation $(\text { EMCS })^{[27]}$ and Interval Monte Carlo simulation (IMCS) ${ }^{[20]}$, have drawn special attentions. The EMCS method is a non-intrusive methods, thus can be applied to any black-box models. However, this method is not applicable for problems with high-dimensional uncertain distribution parameters due to the large estimation errors. The IMCS method is an intrusive method, which may require, e.g., interval finite element analysis, for each interval sample. Besides, the sampling techniques developed in the context of precise probability model have also been extended to the case of imprecise probability models. For example, the importance sampling and SS methods have been extended to estimate the failure probability function ${ }^{[22]}$, which takes the similar idea with the EMCS method; the SS procedure has been extended to estimate the failure probability bounds ${ }^{[30]}$, in which, a large number of response function calls are need for solving the corresponding optimization methods.

Estimating the failure probability functions is more general than calculating the failure probability bounds since that, with it, the probability bounds can be easily computed without calling the response functions, and the failure probability functions also provide basis for further performing sensitivity analysis ${ }^{[31]}$ and/or uncertainty-based design optimization ${ }^{[14][22]}$. In the companion paper ${ }^{[1]}$, based on precise stochastic simulation and high-dimensional model representation (HDMR), a general methodology framework, denoted as imprecise stochastic simulation, has been devised for efficiently propagating the imprecise probability models. This framework consists of a set of new methods which have been proved to be effective for practical applications, and can provide a good balance between local and global performances with estimation errors being properly assessed. In this paper, this new framework will be extended to estimate the failure probability functions subjected to rare failure events. To achieve this target, we firstly combine the imprecise stochastic simulation with the active learning procedure so as to reduce the computational costs. Then, for rare failure events, two SS based sampling techniques, denoted as "LESS (local extended SS)-cut-HDMR" and "GESS (global extended SS)-RSHDMR" methods, are developed with estimation errors being assessed by sensitivity indices and statistical properties of the estimators. Both methods are also further improved by active learning procedure. These new developments substantially enrich the imprecise stochastic simulation framework.

The rest of this paper is organized as follows. Section 2 reviews the imprecise stochastic simulation 
framework for estimating the failure probability functions. Section 3 introduces the active learning method to reduce the computational cost of the imprecise stochastic simulation procedures. Section 4 devises two new imprecise stochastic simulation procedures based on subset simulation. Section 5 introduces the active learning procedure to improve the two methods proposed in section 4 so as to further reduce the computational costs. Three test examples are introduced in section 6 to demonstrate the performance of the proposed method, and section 7 presents some discussions and conclusions to this work.

\section{Imprecise stochastic simulation}

In the companion paper, we have introduced two imprecise stochastic simulation procedures, i.e., LEMCS-cut-HDMR and GEMCS-RS-HDMR, for efficiently estimating the probabilistic response functions ${ }^{[1]}$. Now we briefly review them for estimating the failure probability functions.

Following the notations in the companion paper, denote by $y=g(\boldsymbol{x})$ the limit state function (also termed as $g$-function) with $\boldsymbol{x}=\left(x_{1}, x_{2}, \ldots, x_{n}\right)^{T}$ being the $n$-dimensional random input variables and $y$ indicating the response of interest. Let $f_{X}(\boldsymbol{x} \mid \boldsymbol{\theta})$ denote the joint PDF of $\boldsymbol{x}$ conditional on $\boldsymbol{\theta}$, where $\boldsymbol{\theta}=\left(\theta_{1}, \theta_{2}, \ldots, \theta_{d}\right)^{T}$ refers to the uncertain distribution parameters with assumed joint PDF $f_{\boldsymbol{\Theta}}(\boldsymbol{\theta})=\prod_{i=1}^{d} f_{\Theta_{i}}\left(\theta_{i}\right)$. Then the joint PDF of $\boldsymbol{x}$ and $\boldsymbol{\theta}$ is derived as $f_{\mathbf{x}, \boldsymbol{\Theta}}(\boldsymbol{x}, \boldsymbol{\theta})=f_{\mathbf{X}}(\boldsymbol{x} \mid \boldsymbol{\theta}) f_{\boldsymbol{\Theta}}(\boldsymbol{\theta})$.

We assume that the failure happens when $y<0$, and the failure domain is formulated as $F=\{\boldsymbol{x}: g(\boldsymbol{x})<0\}$. The indicator function of $F$ is formulated by $I_{F}(\boldsymbol{x})=1$ if $\boldsymbol{x} \in F$, and else $I_{F}(\boldsymbol{x})=0$. Then the failure probability function is expressed as:

$$
\mathrm{P}_{f}(\boldsymbol{\theta})=\int_{\mathbf{R}^{n}} I_{F}(\boldsymbol{x}) f_{X}(\boldsymbol{x} \mid \boldsymbol{\theta}) \mathrm{d} \boldsymbol{x}
$$

Based on the HDMR decomposition, the failure probability function can be decomposed as the sum of component functions of increasing orders, i.e.,

$$
\mathrm{P}_{f}(\boldsymbol{\theta})=\mathrm{P}_{f, 0}+\sum_{i=1}^{d} \mathrm{P}_{f, i}\left(\theta_{i}\right)+\sum_{1 \leq i<j \leq d} \mathrm{P}_{f, i j}\left(\boldsymbol{\theta}_{i j}\right)+\ldots+\mathrm{P}_{f, 12 \ldots d}(\boldsymbol{\theta})
$$

If the cut-HDMR decomposition is performed, the failure probability is expended at a fixed point $\boldsymbol{\theta}^{*}$, and the components in the right side of Eq. (2) are given as ${ }^{[1]}$ :

$$
\left\{\begin{array}{l}
\mathrm{P}_{f, \mathrm{cut}, 0}=\mathrm{P}_{f}\left(\boldsymbol{\theta}^{*}\right) \\
\mathrm{P}_{f, \mathrm{cut}, i}\left(\theta_{i}\right)=\mathrm{P}_{f}\left(\theta_{i}, \boldsymbol{\theta}_{-i}^{*}\right)-\mathrm{P}_{f, \mathrm{cut}, 0} \\
\mathrm{P}_{f, \mathrm{cut}, i j}\left(\boldsymbol{\theta}_{i j}\right)=\mathrm{P}_{f}\left(\boldsymbol{\theta}_{i j}, \boldsymbol{\theta}_{-i j}^{*}\right)-\mathrm{P}_{f, \mathrm{cut}, i}\left(\theta_{i}\right)-\mathrm{P}_{f, \mathrm{cut}, j}\left(\theta_{j}\right)-\mathrm{P}_{f, \mathrm{cut}, 0}
\end{array}\right.
$$

where $\boldsymbol{\theta}^{*}$ can be chosen as the mean values of $\boldsymbol{\theta}$ or other points around which one intend to estimate the failure probability function more accurately, $\theta_{i j}$ refers to the two-dimensional vector consists of $\theta_{i}$ and $\theta_{j}, \boldsymbol{\theta}_{-i}^{*}$ indicates the vector containing all elements of $\boldsymbol{\theta}^{*}$ but $\theta_{i}^{*}$.

If the RS-HDMR decomposition is used, the components in Eq. (2) are formulated as ${ }^{[1]}$ : 


$$
\left\{\begin{array}{l}
\mathrm{P}_{f, \mathrm{RS}, 0}=\operatorname{Exp}_{\boldsymbol{\Theta}}\left[\mathrm{P}_{f}(\boldsymbol{\theta})\right] \\
\mathrm{P}_{f, \mathrm{RS}, i}\left(\theta_{i}\right)=\operatorname{Exp}_{\boldsymbol{\Theta}_{-i}}\left[\mathrm{P}_{f}(\boldsymbol{\theta}) \mid \theta_{i}\right]-\mathrm{P}_{f, \mathrm{RS}, 0} \\
\mathrm{P}_{f, \mathrm{RS}, i j}\left(\boldsymbol{\theta}_{i j}\right)=\operatorname{Exp}_{\boldsymbol{\Theta}_{-i j}}\left[\mathrm{P}_{f}(\boldsymbol{\theta}) \mid \boldsymbol{\theta}_{i j}\right]-\mathrm{P}_{f, \mathrm{RS}, i}\left(\theta_{i}\right)-\mathrm{P}_{f, \mathrm{RS}, j}\left(\theta_{j}\right)-\mathrm{P}_{f, \mathrm{RS}, 0}
\end{array}\right.
$$

where $\operatorname{Exp}_{\boldsymbol{\Theta}}[\cdot]$ indicates the expectation operator w.r.t. $\boldsymbol{\theta}, \operatorname{Exp}_{\boldsymbol{\Theta}_{-i}}\left[\bullet \mid \theta_{i}\right]$ and $\operatorname{Exp}_{\boldsymbol{\Theta}_{-i j}}\left[\bullet \mid \boldsymbol{\theta}_{i j}\right]$ refer to the conditional expectation operators w.r.t. $\boldsymbol{\theta}_{-i}$ and $\boldsymbol{\theta}_{-i j}$ respectively. The mathematical properties of the component functions in Eqs. (3) and (4) can be found in the companion paper.

Then, given a set of samples $\boldsymbol{x}^{(k)}(k=1,2, \ldots, N)$ following $f_{X}\left(\boldsymbol{x} \mid \boldsymbol{\theta}^{*}\right)$, the unbiased estimators of the cut-HDMR component functions in Eq. (3) are derived as:

$$
\left\{\begin{array}{l}
\hat{\mathrm{P}}_{f, \mathrm{cu}, 0}=\frac{1}{N} \sum_{k=1}^{N} I_{F}\left(\boldsymbol{x}^{(k)}\right) \\
\hat{\mathrm{P}}_{f, \mathrm{cut}, i}\left(\theta_{i}\right)=\frac{1}{N} \sum_{k=1}^{N} I_{F}\left(\boldsymbol{x}^{(k)}\right) r_{\mathrm{cut}, i}\left(\boldsymbol{x}^{(k)} \mid \theta_{i}, \boldsymbol{\theta}^{*}\right) \\
\hat{\mathrm{P}}_{f, \mathrm{cut}, i j}\left(\boldsymbol{\theta}_{i j}\right)=\frac{1}{N} \sum_{k=1}^{N} I_{F}\left(\boldsymbol{x}^{(k)}\right) r_{\mathrm{cu}, i j}\left(\boldsymbol{x}^{(k)} \mid \boldsymbol{\theta}_{i j}, \boldsymbol{\theta}^{*}\right)
\end{array}\right.
$$

where $r_{\text {cut }, i}$ and $r_{\text {cut }, j i}$ are defined in the companion paper. Given a set of joint samples $\left(\boldsymbol{x}^{(k)}, \boldsymbol{\theta}^{(k)}\right)$ $(k=1,2, \ldots, N)$ generated by $f_{X, \boldsymbol{\Theta}}(\boldsymbol{x}, \boldsymbol{\theta})$, the unbiased estimators for the RS-HDMR component functions in Eq. (4) are derived as:

$$
\left\{\begin{array}{l}
\hat{\mathrm{P}}_{f, \mathrm{RS}, 0}=\frac{1}{N} \sum_{k=1}^{N} I_{F}\left(\boldsymbol{x}^{(k)}\right) \\
\hat{\mathrm{P}}_{f, \mathrm{RS}, i}\left(\theta_{i}\right)=\frac{1}{N} \sum_{k=1}^{N} I_{F}\left(\boldsymbol{x}^{(k)}\right) r_{\mathrm{RS}, i}\left(\boldsymbol{x}^{(k)} \mid \theta_{i}, \boldsymbol{\theta}^{(k)}\right) \\
\hat{\mathrm{P}}_{f, \mathrm{RS}, i j}\left(\boldsymbol{\theta}_{i j}\right)=\frac{1}{N} \sum_{k=1}^{N} I_{F}\left(\boldsymbol{x}^{(k)}\right) r_{\mathrm{RS}, i j}\left(\boldsymbol{x}^{(k)} \mid \boldsymbol{\theta}_{i j}, \boldsymbol{\theta}^{(k)}\right)
\end{array}\right.
$$

where $r_{\mathrm{RS}, i}$ and $r_{\mathrm{RS}, i j}$ are defined in the companion paper. The variances of the estimators can be easily derived and one can refer to the companion paper for details.

For measuring the relative importance of each component function, two sensitivity indices have been introduced in the mate paper. The one for the cut-HDMR component function $\mathrm{P}_{f, \mathrm{cut}, i_{1} i_{2} \ldots i_{s}}\left(\boldsymbol{\theta}_{i_{i} i_{2} \ldots i_{s}}\right)$ is defined as:

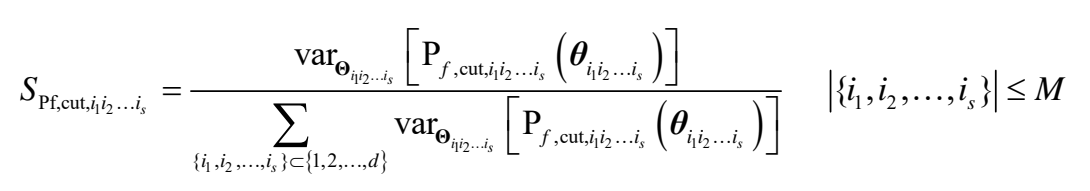

where $\operatorname{var}_{\boldsymbol{\Theta}_{i 12 . . . s}}[\cdot]$ indicates the variance operator w.r.t. $\boldsymbol{\theta}_{i_{i_{1}} \ldots i_{s}}$, and $M$ refers to the highest order under consideration. If this sensitivity index is close to zero, then the corresponding component function must be non-influential, and thus can be eliminated.

The sensitivity index for the RS-HDMR component function $\mathrm{P}_{f, \mathrm{RS}, i_{1} i_{2} \ldots i_{s}}\left(\boldsymbol{\theta}_{i_{i} i_{2} \ldots i_{s}}\right)$ is defined by: 


$$
S_{\mathrm{Pf}, \mathrm{RS}, i_{1} i_{2} \ldots, i_{s}}=\frac{\operatorname{var}_{\boldsymbol{\Theta}_{i i_{2}, \ldots, i_{s}}}\left[\mathrm{P}_{f, \mathrm{RS}, i_{1} i_{2} \ldots i_{s}}\left(\boldsymbol{\theta}_{i_{1} i_{2} \ldots i_{s}}\right)\right]}{\operatorname{var}_{\boldsymbol{\Theta}}\left[\mathrm{P}_{f}(\boldsymbol{\theta})\right]}
$$

Eq. (8) is exactly the well-known Sobol' index ${ }^{[27]-[29]}$. Similarly, if it is close to zero, the corresponding component functions can be neglected. Due to the mutually orthogonal property of the RS-HDMR component functions, the Sobol' index also quantifies the individual and interaction contributions of the epistemic uncertainty of each input variable to that of the failure probability. These information can also be valuable in practical applications since they inform the analysts the most economical way of reducing the epistemic uncertainty presented in the estimation of failure probability. Both sensitivity indices in Eqs. (7) and (8) can be computed by numerical integration procedures or sampling procedures without extra running of the model, and one can refer to the mate paper for detail.

The above procedure can be simply extended to structures with multiple failure modes and/or timevariant excitations. However, these two methods are commonly computationally expensive especially for rare failure events. In the next section, the active learning procedure is introduced for reducing the computational cost.

\section{Improvements of imprecise stochastic simulation by active learning}

The active learning procedures based on, e.g., Kriging surrogate model, have long been used for improving the efficiency of reliability analysis ${ }^{[13]}$. These methods aim at adaptively approximating the $g$-function with one or multiple sets of samples (called sample pools) based on the principle that the signs of the $g$-function at these sample points can be correctly identified, and then estimating the failure probability based on those predicted signs. Based on this idea, as long as the signs of the $g$-function at the sample points used in the LEMCS-cut-HDMR and GEMCS-RS-HDMR procedures can be accurately identified by an active learning procedure, then the failure probability functions can also be effectively estimated without extra calls of g-function. We denote these two active learning procedures as "AKLEMCS-cut-HDMR" and "AK-GEMCS-RS-HDMR" respectively, where "AK" indicates "active learning Kriging".

Take the AK-LEMC-cut-HDMR procedure as an example, the algorithm steps are given as follows.

Step A.1: Generate a sample pool $\mathbf{S}=\left\{\boldsymbol{x}^{(k)}: k=1,2, \ldots, N\right\}$ following $f_{\boldsymbol{X}}\left(\boldsymbol{x} \mid \boldsymbol{\theta}^{*}\right)$. Randomly select $N_{0}$ (e.g., $N_{0}=12$ ) samples from $\mathbf{S}$, and estimate the corresponding $g$-function values. Attribute these $N_{0}$ samples to the training sample set $\mathbf{S}_{T}$.

Step A.2: Train the Kriging surrogate model with $\mathbf{S}_{T}$.

Step A.3: Predict the g-function values $\mu_{g}\left(\boldsymbol{x}^{(k)}\right)$ and the corresponding mean square error $\sigma_{g}^{2}\left(\boldsymbol{x}^{(k)}\right)$ for all the non-training samples contained in $\mathbf{S}$ based on the obtained Kriging model, and judge whether the sign of the $g$-function at each sample point is correctly identified with the principle that $\min _{k=1}^{N} U_{k}>U_{0}$, where $U_{k}=\left|\mu_{g}\left(\boldsymbol{x}^{(k)}\right)\right| / \sigma_{g}\left(\boldsymbol{x}^{(k)}\right)$ and $U_{0}$ is a threshold commonly set to be 2. If it is satisfied, go to Step A.4; Else, find the non-training sample with the smallest $U$ value, compute the corresponding $g$-function value, add this sample into the training sample set $\mathbf{S}_{T}$, and go to Step A.2. 
Step A.4: As the sign of the $g$-function for each input sample contained in $\mathbf{S}$ is correctly identified by the Kriging model, these samples as well as the predicted signs are used for estimating the cutHDMR component functions as well as the corresponding estimators' variances based on Eq. (5).

As the Kriging surrogate model assumes that the $g$-function is a Gaussian random field in the space of the input variables, the value of $\Phi\left(-U_{k}\right)$ in Step 3 is in fact the probability of making a wrong judgement on the sign of $g\left(\boldsymbol{x}^{(k)}\right)$, where $\Phi(\bullet)$ indicates the cumulative distribution function of standard Gaussian distribution. If we let $U_{0}=2$, then $\min _{k=1}^{N} U_{k}>U_{0}$ indicates that the probability of wrongly specifying the sign of each sample is less than $\Phi(-2)=0.023^{[13]}$. The $U$-function is called learning function. One can also use the other learning functions such as the expected feasibility function $(\mathrm{EFF})^{[32]}$. The AK-GEMCS-RS-HDMR procedure employs the same active learning procedure, which is omitted for clarity.

Depending on practical experience, the required population of the sample pool in the above procedure is approximately $(50 \sim 100) / \mathrm{P}_{f, \text { cut }, 0}$, thus for problems with very small $\mathrm{P}_{f, \text { cut, },}$ values (e.g., 1e-6), the sampling procedures introduced in section 2 and the active learning procedures introduced in this section are all inapplicable. For this type of problems, we introduce the extended subset simulation in the next section.

\section{Extended subset simulation}

For structures with rare failure events, the SS has been one of the most popular methods for failure probability estimation in the context of precise probability model. Here, we propose two new imprecise stochastic simulation procedures, denoted as LESS-cut-HDMR and GESS-RS-HDMR, for estimating the failure probability functions subjected to rare failure events.

\subsection{The LESS-cut-HDMR procedure}

The classical SS procedure estimates the small failure probability by introducing a set of intermediate shrinking failure domains $F_{1} \supset F_{2} \supset \ldots \supset F_{m}=F$ and expressing the small failure probability as the product of a set of large conditional probabilities, i.e., $\mathrm{P}_{f, \mathrm{cut}, 0}=\operatorname{Pr}\left(F_{1}\right) \prod_{q=2}^{m} \operatorname{Pr}\left(F_{q} \mid F_{q-1}\right)$, where $F_{q}=\left\{\boldsymbol{x}: g(\boldsymbol{x})<b_{q}\right\}$ and $b_{1}>b_{2}>\ldots>b_{m}=0$. Suppose $\boldsymbol{\theta}$ is fixed at $\boldsymbol{\theta}^{*}$, then given the sampling PDF $f_{X}\left(\boldsymbol{x} \mid \boldsymbol{\theta}^{*}\right)$, the sample size $N$ and the intermediate probability $p_{0}$ (commonly set to be $0.1 \sim 0.3$ ), the SS procedure for estimating the constant cut-HDMR component $\mathrm{P}_{f, \mathrm{cut}, 0}$ is summarized as follows [11].

Step B.1: Generate a MC sample set $\mathbf{S}_{1}=\left\{\boldsymbol{x}_{1}^{(k)}: k=1,2, \ldots, N\right\}$ following $f_{X}\left(\boldsymbol{x} \mid \boldsymbol{\theta}^{*}\right)$, and compute the corresponding values $y_{1}^{(k)}$ of the $g$-function. Let $q=1$.

Step B.2: Sort the value of $y_{q}^{(k)}$ with $k=1,2, \ldots, N$ in ascending order, and set $b_{q}$ as the $\left\lceil p_{0} N\right\rceil$ th sorted $g$-function value. If $b_{q}<0$, go to Step B.4; Else, define the $q$-th intermediate failure surface as $F_{q}=\left\{\boldsymbol{x}: g(\boldsymbol{x})<b_{q}\right\} \quad$ and the corresponding failure sample set as 
$\mathbf{S}_{F q}=\left\{\boldsymbol{x}_{q}^{(k)}: \boldsymbol{x}_{q}^{(k)} \in \mathbf{S}_{q} \cap F_{q}\right\}$, go to Step B.3.

Step B.3: Let $q=q+1$. By setting each element of $\mathbf{S}_{F(q-1)}$ as a starting point, create a conditional sample set $\mathbf{S}_{q}=\left\{\boldsymbol{x}_{q}^{(k)}: k=1,2, \ldots, N\right\}$ following conditional PDF $f_{X}\left(\boldsymbol{x} \mid F_{q-1}, \boldsymbol{\theta}^{*}\right)$ with any MCMC algorithm ${ }^{[11]}$. Go to Step B.2.

Step B.4: Let $m=q, b_{m}=0$ and $F_{m}=F=\left\{\boldsymbol{x}: g(\boldsymbol{x})<b_{m}\right\}$. The constant cut-HDMR component $\mathrm{P}_{f, \text { cut, } 0}$ is estimated by:

$$
\hat{\mathrm{P}}_{f, \text { cut }, 0}=p_{0}^{m-1} \frac{1}{N} \sum_{k=1}^{m} I_{F}\left(\boldsymbol{x}_{m}^{(k)}\right)
$$

Based on the above MC and MCMC sample pools, all the cut-HDMR component functions in Eq. (3) can be estimated, and the LESS estimators for the first- and the second- order component functions are derived as:

$$
\left\{\begin{array}{l}
\hat{\mathrm{P}}_{f, \text { cut }, i}\left(\theta_{i}\right)=p_{0}^{m-1}\left[\frac{1}{N} \sum_{k=1}^{N} I_{F}\left(\boldsymbol{x}_{m}^{(k)}\right) r_{\text {cut }, i}\left(\boldsymbol{x}_{m}^{(k)} \mid \theta_{i}, \boldsymbol{\theta}^{*}\right)\right] \\
\hat{\mathrm{P}}_{f, \text { cut }, j i}\left(\boldsymbol{\theta}_{i j}\right)=p_{0}^{m-1}\left[\frac{1}{N} \sum_{k=1}^{N} I_{F}\left(\boldsymbol{x}_{m}^{(k)}\right) r_{\text {cut }, i j}\left(\boldsymbol{x}_{m}^{(k)} \mid \boldsymbol{\theta}_{i j}, \boldsymbol{\theta}^{*}\right)\right]
\end{array}\right.
$$

One can refer to Appendix A for the derivations of the above estimators. The statistical properties of the estimators in Eq. (10) are analogous to that of the classical SS estimator, i.e., the estimators are asymptotically unbiased and the bias is $\mathrm{O}(1 / N)$, which is caused by the correlations between the estimators of the intermediate probabilities ${ }^{[1]}$. Based on the assumption that the estimators of all the intermediate probabilities are mutually independent, the variance of, e.g., the estimator $\hat{\mathrm{P}}_{f, \text { cut }, i}$, can be approximated by ${ }^{[11]}$ :

$$
\operatorname{var}\left[\hat{\mathrm{P}}_{f, \text { cut }, i}\right] \hat{=} \frac{\hat{\mathrm{P}}_{f, \text { cut }, i}^{2-1}}{\hat{P}_{q}^{2}} \sum_{q=1}^{m} \operatorname{var}\left[\hat{P}_{q}\right]+\frac{\hat{\mathrm{P}}_{f, \mathrm{cut}, i}^{2}}{\hat{P}_{m}^{2}\left(\theta_{i}\right)} \operatorname{var}\left[\hat{P}_{m}\left(\theta_{i}\right)\right]
$$

where $\hat{P}_{q}=\sum_{k=1}^{N} I_{F_{q}}\left(\boldsymbol{x}_{q}^{(k)}\right) / N$ and $\hat{P}_{m}\left(\theta_{i}\right)=\sum_{k=1}^{N} I_{F}\left(\boldsymbol{x}_{m}^{(k)}\right) r_{\text {cut }, i}\left(\boldsymbol{x}_{m}^{(k)} \mid \theta_{i}, \boldsymbol{\theta}_{-i}^{*}\right) / N$. Ref.[11] has shown that Eq. (11) commonly provides good approximation to $\operatorname{var}\left[\hat{\mathrm{P}}_{f, \text { cut, }, i}\right]$. In Eq. (11), $\operatorname{var}\left[\hat{P}_{q}\right]$ and $\operatorname{var}\left[\hat{P}_{m}\left(\theta_{i}\right)\right]$ can be approximated by ${ }^{[11]}$ :

$$
\left\{\begin{array}{l}
\operatorname{var}\left[\hat{P}_{q}\right] \triangleq \frac{\hat{P}_{q}-\hat{P}_{q}^{2}}{(N-1)}\left(1+\gamma_{q}\right) \\
\operatorname{var}\left[\hat{P}_{m}\left(\theta_{i}\right)\right]=\frac{1}{N-1}\left[\frac{1}{N} \sum_{k=1}^{N} I_{F}\left(\boldsymbol{x}_{m}^{(k)}\right) r_{\mathrm{cut}, i}^{2}\left(\boldsymbol{x}_{m}^{(k)} \mid \theta_{i}, \boldsymbol{\theta}^{*}\right)-\hat{P}_{m}^{2}\left(\theta_{i}\right)\right]\left[1+\gamma_{m}\right]
\end{array}\right.
$$

where $\gamma_{q}(q=1,2, \ldots, m)$ is a factor related to the correlations of the samples contained in the same Markov chain. In the SS procedure, strong correlations among the MCMC samples generally lead to poor estimations. If the sample correlations are weak, then $\gamma_{q}$ can be neglected.

Similar to the classical SS procedure, for generating the MCMC samples, the variables should be 
better transformed into the independent standard normal space, which can be achieved by Nataf transformation ${ }^{[34]}$ or Rosenblatt transformation ${ }^{[35]}$. Take the independent input variables as an example. Denote the distribution of $x_{i}$ as $F_{X_{i}}\left(x_{i} \mid \boldsymbol{\theta}_{(i)}\right)$ with $\boldsymbol{\theta}_{(i)}$ being the vector of the distribution parameters of $x_{i}$. Then both transformations are formulated as $x_{i}=F_{X_{i}}^{-1}\left(\Phi\left(u_{i}\right) \mid \boldsymbol{\theta}_{(i)}^{*}\right)$ with $\boldsymbol{\theta}_{(i)}$ being fixed at $\boldsymbol{\theta}_{(i)}^{*}$, where $u_{i}$ refers to the standard normal variable related to $x_{i}$. Then the above procedure can be implemented in the independent standard normal space. The difference between the two transformation methods in the dependent case is that, the joint distribution function is required for Rosenblatt transformation, while for Nataf transformation, only the correlation matrix is required. One can refer to Refs. [34] and [35] for details. One should note that, although the subset simulation is implemented in the standard normal space, the values of the ratio functions $r_{\text {cut }, i}$ and $r_{\text {cut }, j i}$ in Eq. (10) should be calculated by transforming the sample back to the $\boldsymbol{x}$-space.

The most widely used MCMC algorithm is the so-called Metropolis-Hastings (M-H) algorithm, which commonly results in low acceptance rate and strong correlations among samples especially in high dimension ${ }^{[33]}$. Thus, many efforts have been made by researchers to improve the acceptance rate of the $\mathrm{M}-\mathrm{H}$ algorithm in the context of SS. One of the most well-known improvements is the modified M-H algorithm, which rejects or accepts the candidate state component by component ${ }^{[11]}$. Other improvements include the M-H algorithm with repeated generation of candidate states ${ }^{[36]}$, the modified $\mathrm{M}-\mathrm{H}$ algorithm with delayed rejection ${ }^{[37]}$, and so on. Recently, a new MCMC algorithm based on imposing a joint normal distribution between the current state and the candidate state has also been developed, and it has been shown to be effective especially in high dimension ${ }^{[38][39]}$. In this work, the modified M-H algorithm is applied.

It is easy to prove that all the estimators of the cut-HDMR component functions in Eqs. (9) and (10) possess the vanishing and mutually orthogonal properties, thus they have good local performance. In the next subsection, we propose the GESS-RS-HDMR procedure.

\subsection{The GESS-RS-HDMR procedure}

In this subsection, the GESS procedure is proposed for efficiently estimating the RS-HDMR component functions defined in Eq. (4). Different with the LESS method which generates the intermediate failure surface in the $n$-dimensional input space of $\boldsymbol{x}$, the GESS method produces the intermediate surfaces in the $(n+d)$-dimensional joint input space of $(\boldsymbol{x}, \boldsymbol{\theta})$, and one does not need to specify a fixed value for $\boldsymbol{\theta}$. We firstly present the GESS procedure for estimating the constant RSHDMR component $\mathrm{P}_{f, \mathrm{RS}, 0}$ as follows ${ }^{[11]}$.

Step C.1: Generate a MC sample pool $\mathbf{S}_{1}=\left\{\left(\boldsymbol{x}_{1}^{(k)}, \theta_{1}^{(k)}\right): k=1,2, \ldots, N\right\}$ following the joint PDF $f_{\mathbf{X}, \boldsymbol{\Theta}}(\boldsymbol{x}, \boldsymbol{\theta})$, and compute the g-function value $y_{1}^{(k)}=g\left(\boldsymbol{x}_{1}^{(k)}\right)$ for $k=1,2, \ldots, N$. Let $q=1$.

Step C.2: Sort the values of $y_{q}^{(k)}$ for $k=1,2, \ldots, N$ in ascending order, and make $b_{q}$ equal to the $\left\lceil p_{0} N\right\rceil$-th sorted g-function value. If $b_{q}<0$, go to Step C.4; Else, denote the $q$-th joint failure domain as $F_{q}=\left\{(\boldsymbol{x}, \boldsymbol{\theta}): g(\boldsymbol{x})<b_{q}\right\}$ and the corresponding failure sample set as 
$\mathbf{S}_{F q}=\left\{\boldsymbol{x}_{q}^{(k)}: \boldsymbol{x}_{q}^{(k)} \in S_{q}\right.$ and $\left.g\left(\boldsymbol{x}_{q}^{(k)}\right)<b_{q}\right\}$, go to Step C.3.

Step C.3: Let $q=q+1$. By setting the sample points contained in $\mathbf{S}_{F q}$ as the starting states, generate a new MCMC sample set $\mathbf{S}_{q}=\left\{\boldsymbol{x}_{q}^{(k)}: k=1,2, \ldots, N\right\}$ following $f_{\boldsymbol{X}, \boldsymbol{\Theta}}\left(\boldsymbol{x}, \boldsymbol{\theta} \mid F_{q-1}\right)$ with any MCMC algorithms ${ }^{[11]}$. Go to Step C.2.

Step C.4: Let $m=q, b_{m}=0$ and $F_{m}=\left\{(\boldsymbol{x}, \boldsymbol{\theta}): g(\boldsymbol{x})<b_{m}\right\}$. The RS-HDMR constant term $\hat{\mathrm{P}}_{f, \mathrm{RS}, 0}$ can then be estimated by

$$
\hat{\mathrm{P}}_{f, \mathrm{RS}, 0}=p_{0}^{m-1}\left[\frac{1}{N} \sum_{k=1}^{m} I_{F}\left(\boldsymbol{x}_{m}^{(k)}\right)\right]
$$

Based on the above MC and MCMC sample pools, the GESS estimators of the first- and secondorder RS-HDMR component functions are derived as:

$$
\left\{\begin{array}{l}
\hat{\mathrm{P}}_{f, \mathrm{RS}, i}\left(\theta_{i}\right)=p_{0}^{m-1}\left[\frac{1}{N} \sum_{k=1}^{N} I_{F}\left(\boldsymbol{x}_{m}^{(k)}\right) r_{\mathrm{RS}, i}\left(\boldsymbol{x}_{m}^{(k)} \mid \theta_{i}, \boldsymbol{\theta}_{m}^{(k)}\right)\right] \\
\hat{\mathrm{P}}_{f, \mathrm{RS}, i j}\left(\boldsymbol{\theta}_{i j}\right)=p_{0}^{m-1}\left[\frac{1}{N} \sum_{k=1}^{N} I_{F}\left(\boldsymbol{x}_{m}^{(k)}\right) r_{\mathrm{RS}, i j}\left(\boldsymbol{x}_{m}^{(k)} \mid \boldsymbol{\theta}_{i j}, \boldsymbol{\theta}_{m}^{(k)}\right)\right]
\end{array}\right.
$$

The derivations of the above estimators are presented in Appendix B. One can similarly generate the GESS estimators for higher order RS-HDMR component functions, and the details are omitted for clarity.

The statistical properties of the above GESS estimators for all the RS-HDMR component functions are similar to those of the LESS estimators of the cut-HDMR component functions, thus we won't repeat them. Both the LESS-cut-HDMR and GESS-RS-HDMR procedures can estimate the failure probability functions efficiently for rare failure events with the same computational cost as the classical SS procedure. However, in practical applications involving complex engineering structures, the computational costs of these two procedures are still unacceptable ${ }^{[16]}$. In the next section, we use the active learning procedure to substantially reduce the number of required g-function calls without loss of accuracy.

\section{Improvements of extended subset simulation by active learning}

In the classical SS procedure, $N \mathrm{~g}$-function evaluations are required for generating each intermediate failure surface, thus the total number of required $g$-function calls is $m N$. Further, since $p_{0}$ is commonly set to be $0.1 \sim 0.3$, for rare failure event, $m$ is commonly large for rare failure events. Both factors lead to the high computational cost of the classical SS procedure, and the same holds for the newly developed LESS-cut-HDMR and GESS-RS-HDMR procedures. In our previous work ${ }^{[40]}$, an active learning Kriging procedure called AK-MCMC has been developed in the framework of precise probability model for estimating the extremely small failure probability. In this section, we extend this idea to reduce the computational costs of both LESS-cut-HDMR and GESS-RS-HDMR procedures, and the developed methods are denoted as "AK-LESS-cut-HDMR" and "AK-GESS-RS-HDMR" respectively.

Take the AK-LESS-cut-HDMR procedure as an example, the main steps are summarized as follows.

Step D.1: Generate a sample pool $\mathbf{S}_{1}=\left\{\boldsymbol{x}_{1}^{(k)}: k=1,2, \ldots, N\right\}$ following $f_{X}\left(\boldsymbol{x} \mid \boldsymbol{\theta}^{*}\right)$. Randomly select $N_{0}$ samples points from $\mathbf{S}_{1}$ and compute the corresponding g-function values. Attribute these $N_{0}$ samples of $\boldsymbol{x}$ and $y$ to the training data set $\mathbf{S}_{T}$. Let $q=1$. 
Step D.2: Train the Kriging surrogate model with $\mathbf{S}_{T}$.

Step D.3: Predict the $g$-function values for each sample contained in $\mathbf{S}_{q}-\mathbf{S}_{T}$, and compute or update the value of $b_{q}$ such that $\left\lfloor p_{0} N\right\rfloor$ samples is contained in the failure domain $F_{q}=\left\{\boldsymbol{x}: \hat{\boldsymbol{g}}(\boldsymbol{x})<b_{q}\right\}$. If $b_{q}<0$, let $b_{q}=0$. Compute the U-function values $U_{k}=\left|\mu_{g}\left(\boldsymbol{x}^{(k)}\right)-b_{q}\right| / \sigma_{g}\left(\boldsymbol{x}^{(k)}\right)$ for each sample contained in $\mathbf{S}_{q}-\mathbf{S}_{T}$. If $\min U_{k}>U_{0}$, go to Step D.4; else, find the sample in $\mathbf{S}_{q}-\mathbf{S}_{T}$ with the minimum $U$ value, compute the corresponding $g$-function values by calling the true computational model, add this sample point to $\mathbf{S}_{T}$, and go to Step D.2.

Step D.4: If $b_{q}=0$, go to Step D.5; Else, let $q=q+1$, generate a conditional sample pool $\mathbf{S}_{q}=\left\{\boldsymbol{x}_{q}^{(k)}: k=1,2, \ldots, N\right\}$ following conditional PDF $f_{\boldsymbol{X}}\left(\boldsymbol{x} \mid F_{q-1}, \boldsymbol{\theta}^{*}\right)$ with any MCMC algorithm by calling the well-trained Kriging surrogate model instead of the true model, and turn to Step D.3.

Step D.5: Let $m=q$, and estimate the cut-HDMR components by:

$$
\left\{\begin{array}{l}
\hat{\mathrm{P}}_{f, \text { cut }, 0}=\prod_{q=1}^{m}\left[\frac{1}{N} \sum_{k=1}^{N} \hat{I}_{F_{q}}\left(\boldsymbol{x}_{q}^{(k)}\right)\right] \\
\hat{\mathrm{P}}_{f, \text { cut }, i}\left(\theta_{i}\right)=\prod_{q=1}^{m-1}\left[\frac{1}{N} \sum_{k=1}^{N} \hat{I}_{F_{q}}\left(\boldsymbol{x}_{q}^{(k)}\right)\right]\left[\frac{1}{N} \sum_{k=1}^{N} \hat{I}_{F_{m}}\left(\boldsymbol{x}_{m}^{(k)}\right) r_{\mathrm{cut}, i}\left(\boldsymbol{x}_{m}^{(k)} \mid \theta_{i}, \boldsymbol{\theta}^{*}\right)\right] \\
\hat{\mathrm{P}}_{f, \mathrm{cut}, i j}\left(\boldsymbol{\theta}_{i j}\right)=\prod_{q=1}^{m-1}\left[\frac{1}{N} \sum_{k=1}^{N} \hat{I}_{F_{q}}\left(\boldsymbol{x}_{q}^{(k)}\right)\right]\left[\frac{1}{N} \sum_{k=1}^{N} \hat{I}_{F_{m}}\left(\boldsymbol{x}_{m}^{(k)}\right) r_{\mathrm{cut}, i j}\left(\boldsymbol{x}_{m}^{(k)} \mid \boldsymbol{\theta}_{i j}, \boldsymbol{\theta}^{*}\right)\right]
\end{array}\right.
$$

where the hat symbol '^' above the indicator function indicates that the signs of the $g$-function are predicted by the Kriging surrogate models well-trained for approximating the corresponding failure surfaces.

In the above procedure, the intermediate probability may not definitely equal to $p_{0}$ since that the convergence condition only promises that the sign of $g(\boldsymbol{x})-b_{q}$ is accurately predicted, but does not guarantee that each intermediate probability completely converges to $p_{0}$. However, the estimated intermediate probability is usually very close to $p_{0}$. Thus, the former $(m-1)$ intermediate probabilities should be estimated based on the corresponding sample pools and the well-trained Kriging model instead of making them equal to $p_{0}$. In this algorithm, $p_{0}$ is suggested to be $1 \mathrm{e}-3 \sim 1 \mathrm{e}-2$, and $N$ is suggested to be $(50 \sim 100) / p_{0}$. Thus, compared with the LESS-cut-HDMR procedure, the number of required intermediate failure surfaces has been largely reduced. If the real value of $\mathrm{P}_{f, \mathrm{cut}, 0}$ is larger than $p_{0}$, then the above algorithm degrades to the one introduced in section 3.

The AK-GESS-RS-HDMR method can be similarly carried out except that the sample pools $\mathbf{S}_{q}$ should be generated in the $(n+d)$-dimensional joint space of $(\boldsymbol{x}, \boldsymbol{\theta})$ instead of the $n$-dimensional space of $\boldsymbol{x}$. For simplicity, we don't repeat the process. In the next section, we introduce several test examples to illustrate and compare the proposed methods.

\section{Test examples and engineering applications}




\subsection{A toy test example}

We still consider the toy test example introduced in the mate paper [1]. Here, we consider two cases. For case 1, $a$ and $b$ are set to be 3 and 4 respectively, while for case 2, $a$ and $b$ are assumed to be 5 and 6 respectively. The ranges of the distribution parameters are set to be the same with the mate paper.

We firstly implement the LEMCS-cut-HDMR and AK-LEMCS-cut-HDMR procedures for case 1. The expansion point $\boldsymbol{\theta}^{*}$ is assumed to be the mean values, i.e., $\boldsymbol{\theta}^{*}=(0,0,1,1)^{T}$. A same set of $5 \mathrm{e} 4$ samples are used for both methods, and the total number of training samples used in the active learning procedure turns out to be 28 , indicating that the total number of function calls of these two methods are $5 \mathrm{e} 4$ and 28 respectively. We compute the sensitivity indices based on the estimated component functions as well as their SDs, and the results of the first- and second- order sensitivity indices are reported in the Table 1 and Table 2 respectively. It is seen that the sensitivity indices computed by these two methods match well, and their variations as indicated by the superscripts are all small. It is shown in Table 1 and Table 2 that, among the first-order cut-HDMR component functions, only those of $\mu_{1}$ and $\sigma_{1}$ are influential, and among the second-order cut-HDMR component functions, only the one of $\left(\mu_{1}, \sigma_{1}\right)$ is influential. Thus, we display the results of the two influential first-order component functions in Fig. 1, together with the reference results generated by the crude MCS procedure for comparison, and the results of the influential second-order component function are shown in Fig. 2.

Table 1 Results of the sensitivity indices of the first-order cut-HDMR and RS-HDMR component functions for case 1 of the toy test example, where the superscripts indicate the results computed by integrating the corresponding SDs of the estimates of the component functions

\begin{tabular}{|c|c|c|c|c|}
\hline \multirow{2}{*}{ Indices } & \multicolumn{2}{|c|}{$S_{\mathrm{Pf}, \mathrm{cut}, i}$} & \multicolumn{2}{c|}{$S_{\mathrm{Pf}, \mathrm{RS}, i}$} \\
\hline Methods & LEMCS-cut-HDMR & AK-LEMCS-cut-HDMR & GEMCS-RS-HDMR & AK-GEMCS-RS-HDMR \\
\hline Settings & $N=5 \mathrm{e} 4$ & $N=5 \mathrm{e} 4$ & $N=5 \mathrm{e} 4$ & $N=5 \mathrm{e} 4$ \\
\hline$\mu_{1}$ & $.1870^{(.0002)}$ & $.1870^{(.0002)}$ & $.1811^{(.0005)}$ & $.1811^{(.0005)}$ \\
\hline$\mu_{2}$ & $.0130^{(.0001)}$ & $.0130^{(.0001)}$ & $.0148^{(.0001)}$ & $.0148^{(.0001)}$ \\
\hline$\sigma_{1}$ & $.7648^{(.0013)}$ & $.7648^{(.0013)}$ & $.7736^{(.0048)}$ & $.7736^{(.0048)}$ \\
\hline$\sigma_{2}$ & $.0084^{(.0005)}$ & $.0084^{(.0005)}$ & $.0042^{(.0009)}$ & $.0042^{(.0009)}$ \\
\hline $\mathrm{P}_{f, 0}$ & $.0163^{(.0006)}$ & $.0163^{(.0006)}$ & $.0182^{(.0006)}$ & $.0182^{(.0006)}$ \\
\hline$N_{\text {call }}$ & $5 \mathrm{e} 4$ & 28 & $5 \mathrm{e} 4$ & 31 \\
\hline
\end{tabular}

Table 2 Results of the sensitivity indices of the second-order cut-HDMR and RS-HDMR component functions for case 1 of the toy test example

\begin{tabular}{|c|c|c|c|c|}
\hline Indices & \multicolumn{2}{|c|}{$S_{\mathrm{Pf}, \mathrm{cut}, i j}$} & \multicolumn{2}{c|}{$S_{\mathrm{Pf}, \mathrm{RS}, i j}$} \\
\hline Methods & LEMCS-cut-HDMR & AK-LEMCS-cut-HDMR & GEMCS-RS-HDMR & AK-GEMCS-RS-HDMR \\
\hline$\left(\mu_{1}, \mu_{2}\right)$ & $.0006^{(.0000)}$ & $.0006^{(.0000)}$ & $.0005^{(.0000)}$ & $.0005^{(.0000)}$ \\
\hline$\left(\mu_{1}, \sigma_{1}\right)$ & $.0233^{(.0001)}$ & $.0233^{(.0001)}$ & $.0233^{(.0007)}$ & $.0233^{(.0007)}$ \\
\hline$\left(\mu_{1}, \sigma_{2}\right)$ & $.0000^{(.0000)}$ & $.0000^{(.0000)}$ & $.0000^{(.0001)}$ & $.0000^{(.0001)}$ \\
\hline$\left(\mu_{2}, \sigma_{1}\right)$ & $.0018^{(.0001)}$ & $.0018^{(.0001)}$ & $.0015^{(.0001)}$ & $.0015^{(.0001)}$ \\
\hline
\end{tabular}




\begin{tabular}{|c|c|c|c|c|}
\hline$\left(\mu_{2}, \sigma_{2}\right)$ & $.0008^{(.0000)}$ & $.0008^{(.0000)}$ & $.0005^{(.0002)}$ & $.0005^{(.0002)}$ \\
\hline$\left(\sigma_{2}, \sigma_{2}\right)$ & $.0003^{(.0000)}$ & $.0003^{(.0000)}$ & $.0004^{(.0002)}$ & $.0004^{(.0002)}$ \\
\hline
\end{tabular}

The constant cut-HDMR components computed by the LEMCS-cut-HDMR and AK-LEMCS-cutHDMR methods are both 0.0163 , with SDs being both $5.69 \mathrm{e}-4$, and the reference solution computed by the crude MCS procedure with $1 \mathrm{e} 7$ samples is 0.0161 , with SD being 3.98e-5, indicating that the constant component are accurately estimated by both methods. Fig. 1 shows that the two influential cut-HDMR first-order component functions are accurately estimated by both methods when compared with the reference solutions, and the results are also robust due to the very small SDs. It is seen that, both component functions equal to zero at the expansion points $\boldsymbol{\theta}^{*}$, indicating that the corresponding estimators possess the vanishing property. It is seen from Fig. 2 that the influential second-order cutHDMR component function computed by these two methods coincide exactly. As the estimators are unbiased and the SDs are small enough, we conclude that this component function is accurately and robustly estimated by both methods. One should note that all the first- and second- order cut-HDMR component functions are estimated with one set of samples, and higher order component functions can also be computed with this set of samples.

(a)

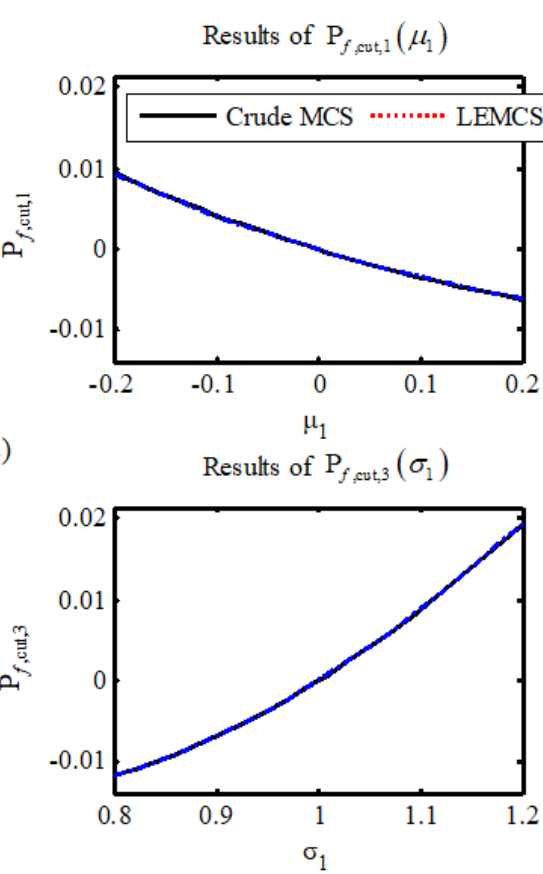

(b)

$\mathrm{x} 10^{-4} \quad \mathrm{SDs}$ of $\mathrm{P}_{f, \mathrm{cut}, 1}\left(\mu_{1}\right)$

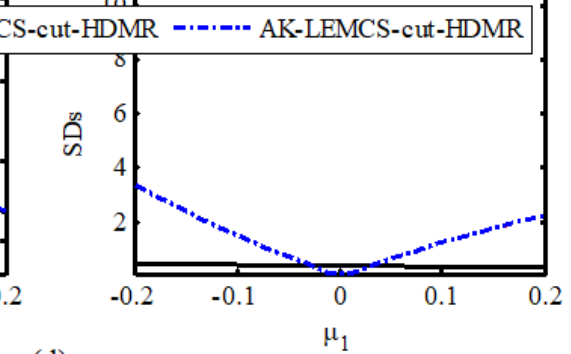

(d)

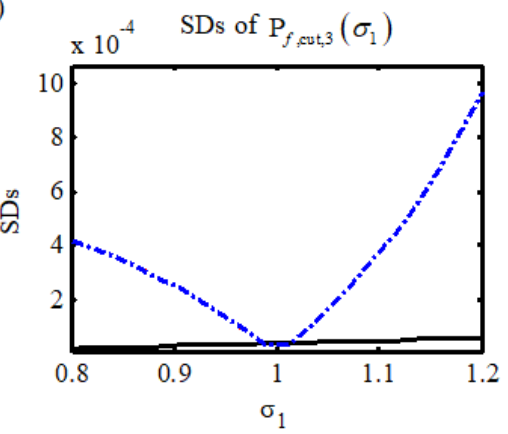

Fig. 1 Results of the two influential first-order cut-HDMR component functions for case 1 of the toy test example, where (a) and (c) indicates the estimated results, (b) and (d) refer to the SDs. 
(a)

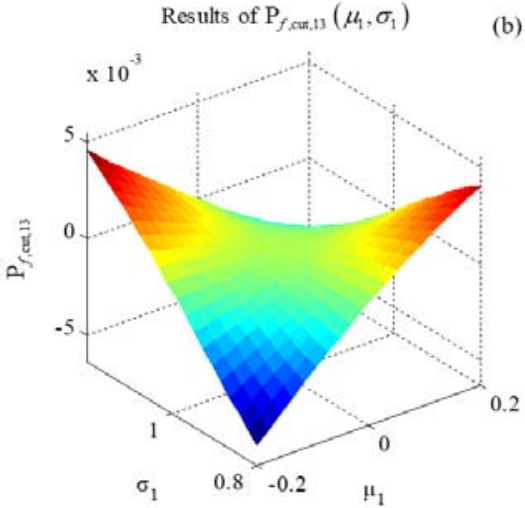

b)

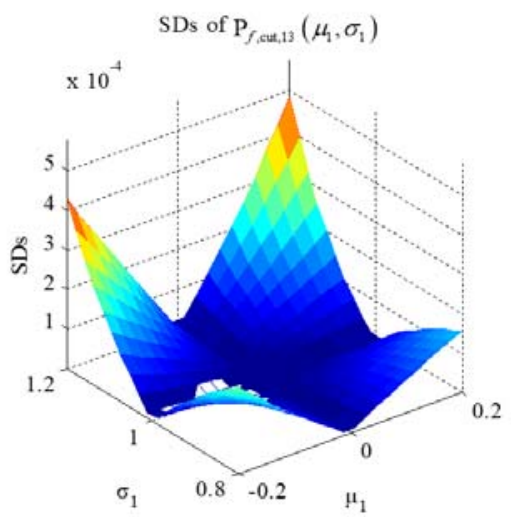

Fig. 2 Results of the influential second-order cut-HDMR component function, where the mesh surfaces indicate the results obtained by the LEMCS-cut-HDMR method, and the smooth surfaces without mesh indicate the results computed by the AK-LEMCS-cut-HDMR method. In this figure, these two surfaces coincide exactly.

We now compute the RS-HDMR component functions for case 1 by the GEMCS-RS-HDMR and AK-GEMCS-RS-HDMR methods with the same set of 1 e5 samples. It is found that $30 \mathrm{~g}$-function calls are consumed in the AK-GEMCS-RS-HDMR method, indicating that the total number of function calls of these two methods are 5e 4 and 30 respectively. The constant RS-HDMR components computed by both methods are 0.0189 with SDs being 6.10e- 4 , and the reference result computed by the crude MCS with 1e7 samples is 0.0183 with SD being 4.24e-5. Thus, the constant component are accurately computed by both methods. The corresponding sensitivity indices are computed based on the estimated component functions, and the standard errors are computed by integrating the SDs of the estimates. The results are displayed in the right half of Table 1 and Table 2. As can be seen, all the sensitivity indices are accurately computed. From the sensitivity indices, we can draw the conclusions that, only the firstorder RS-HDMR component functions of $\mu_{1}$ and $\sigma_{1}$, and the second-order RS-HDMR component function of $\left(\mu_{1}, \sigma_{1}\right)$ are influential, and their results are displayed in Fig. 3 and Fig. 4. As can be seen, all the three RS-HDMR component functions are effectively estimated. Thus, the failure probability functions must be accurately computed. 
(a)

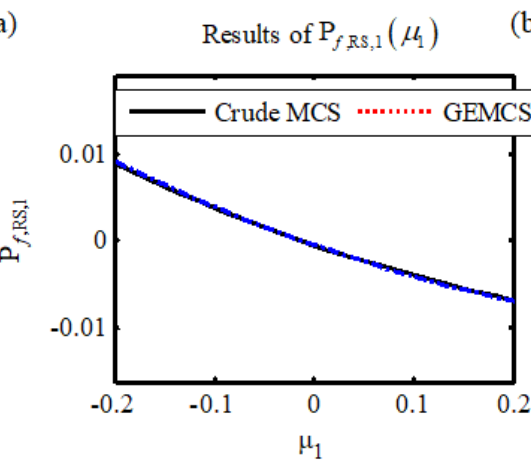

(b) $\mathrm{x} 10^{4} \quad \mathrm{SDs}$ of $\mathrm{P}_{f, R S, 1}\left(\mu_{1}\right)$

(c)

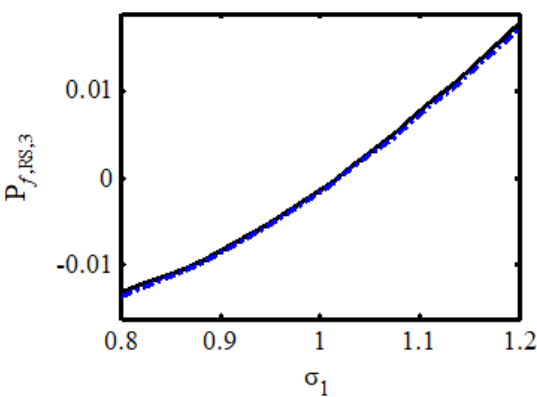

(d)
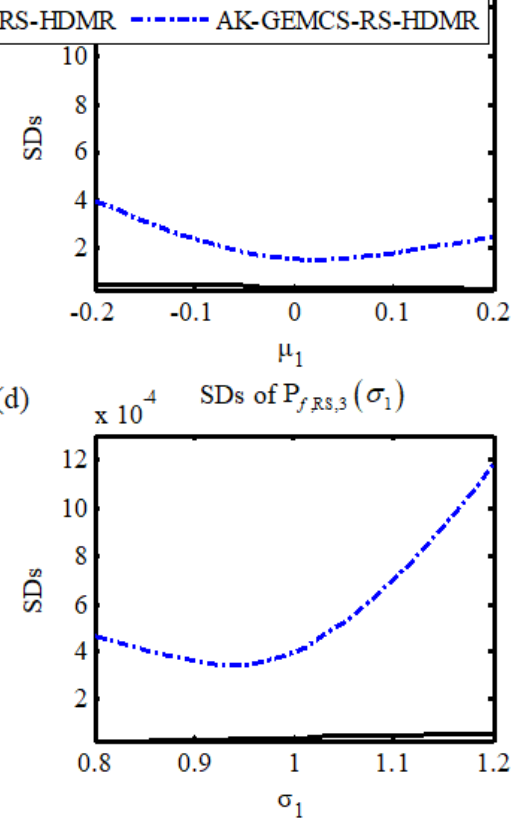

Fig. 3 Results of the two influential first-order RS-HDMR component functions for case 1 of the toy test example, where (a) and (c) indicate the estimations, and (b) and (d) refer to the corresponding SDs.

(a)

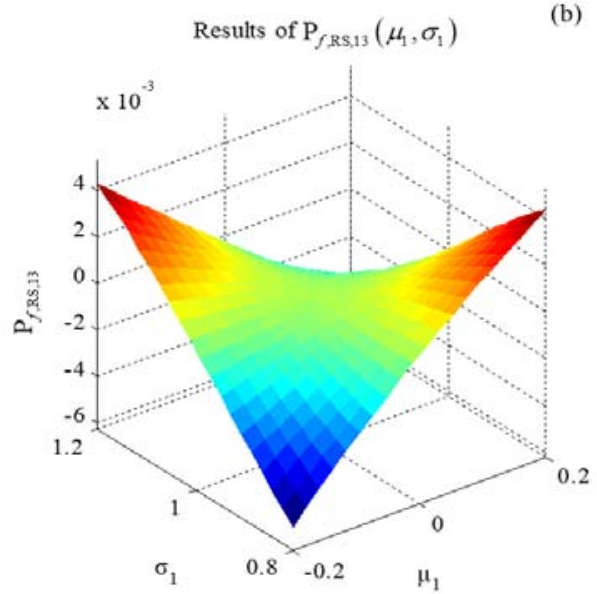

(b)

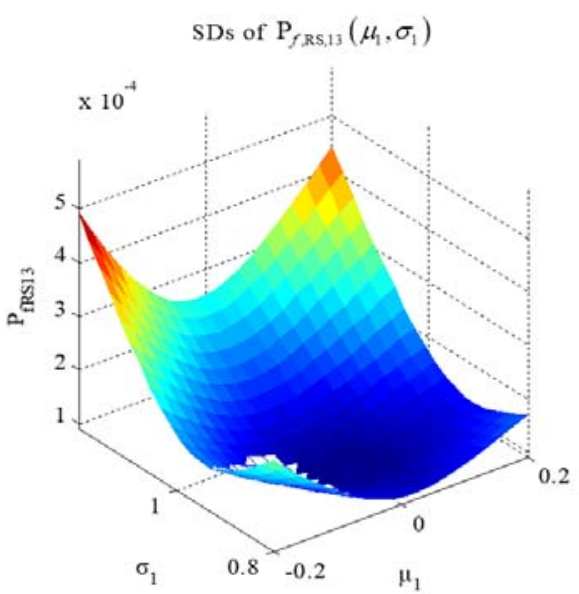

Fig. 4 Results of the influential second-order RS-HDMR component function for case 1 of the toy test example. The results computed by the two methods coincide exactly.

Next we consider case 2 . We firstly estimate the cut-HDMR component functions by the LESS-cutHDMR and AK-LESS-cut-HDMR methods. For LESS-cut-HDMR method, the sample size $N$ in each level is set to be $1 \mathrm{e} 4, p_{0}$ is set to be 0.1 , while for AK-LESS-cut-HDMR method, $N$ is set to be $1 \mathrm{e} 5$ and $p_{0}$ is set to be 1e-3. The LESS-cut-HDMR method produces five intermediate failure surfaces with failure thresholds being $0.8285,0.5895,0.3573,0.1175$ and 0 , while the AK-LESS-cut-HDMR procedure adaptively produces two intermediate failure surfaces with the failure thresholds being 0.3488 and 0 . For adaptively approximating the two intermediate failure surfaces by Kriging surrogate model, $34 \mathrm{~g}$ function calls have been consumed, thus the total number of function calls of these two methods are $5 \mathrm{e} 4$ 
and 34 respectively.

The constant cut-HDMR component estimated by the two methods are $3.59 \mathrm{e}-5$ and $3.97 \mathrm{e}-5$ with SDs being 4.41e-6 and 7.76e-6 respectively, while the reference solution estimated by the crude MCS is 3.55e5 with SD being $1.88 \mathrm{e}-6$. Thus, the constant component is accurately estimated by both methods. With the same sets of MC and MCMC sample pools, the sensitivity indices are computed by the two methods, and the results are displayed in Table 3 and Table 4. As can be seen, among the first-order cut-HDMR component functions, only those of $\sigma_{1}$ and $\sigma_{2}$ are influential, and among the six second order component functions, only those of $\left(\mu_{1}, \sigma_{1}\right)$ and $\left(\mu_{2}, \sigma_{2}\right)$ are important, thus we only display the results of these four cut-HDMR component functions in Fig. 5 and Fig. 6. As can be seen, all the components are accurately and robustly estimated by both the LESS-cut-HDMR and AK-LESS-cutHDMR methods.

Next, we estimate the RS-HDMR components by the GESS-RS-HDMR and AK-GESS-RS-HDMR methods with sensitivity indices results shown in the last two columns of Table 3 and Table 4 . As can be seen, the GESS-RS-HDMR procedure adaptively produces four intermediate failure surfaces with failure thresholds being $0.8310,0.5623,0.2834$ and 0 respectively, while the AK-GESS-RS-HDMR procedure adaptively generates two intermediate failure surfaces with the failure thresholds being 0.5673 and 0 respectively. The constant RS-HDMR components estimated by the two methods are 1.2030e-4 and 1.3187e-4 with SDs being 1.1022e-5 and 5.4984e-6, and the reference solution computed by the crude MCS procedure with 1e7 samples is $1.2020 \mathrm{e}-4$ with SD being 3.4668e-6. Thus the constant RS-HDMR component is accurately and robustly estimated by both methods. From the sensitivity indices, it is seen that, all the four first-order component functions are influential, and among the six second-order component functions, only those of $\left(\mu_{1}, \sigma_{1}\right)$ and $\left(\mu_{2}, \sigma_{2}\right)$ are influential. Thus, the six influential component functions are sufficient for approximating the failure probability function, and their results are shown in Fig. 7 and Fig. 8. As can be seen, all the components are effectively estimated.

Table 3 Results of the sensitivity indices of the first-order cut-HDMR and RS-HDMR component functions for case 2 of the toy test example, where " $b_{i}$ values" refers to the intermediate failure thresholds, $\mathrm{P}_{f, 0}$ indicates the constant HDMR components, and $N_{\text {call }}$ is the total number of function calls of the corresponding method.

\begin{tabular}{|c|c|c|c|c|}
\hline Indices & \multicolumn{2}{|c|}{$S_{\mathrm{Pf}, \mathrm{cut}, i}$} & \multicolumn{2}{|c|}{$S_{\mathrm{Pf}, \mathrm{RS}, i}$} \\
\hline Methods & LESS-cut-HDMR & AK-LESS-cut-HDMR & GESS-RS-HDMR & AK-GESS-RS-HDMR \\
\hline Settings & $p_{0}=0.1, N=1 \mathrm{e} 4$ & $p_{0}=1 \mathrm{e}-3, N=1 \mathrm{e} 5$ & $p_{0}=0.1, N=1 \mathrm{e} 4$ & $p_{0}=1 \mathrm{e}-3, N=1 \mathrm{e} 5$ \\
\hline$\mu_{1}$ & $.0136^{(.0001)}$ & $.0133^{(.0001)}$ & $.0669^{(.0003)}$ & $.0852^{(.0003)}$ \\
\hline$\mu_{2}$ & $.0059^{(.0000)}$ & $.0056^{(.0001)}$ & $.0380^{(.0002)}$ & $.0672^{(.0003)}$ \\
\hline$\sigma_{1}$ & $.6122^{(.0024)}$ & $.6339_{(.0066)}$ & $.4554^{(.0056)}$ & $.4155^{(.0026)}$. \\
\hline$\sigma_{2}$ & $.2403^{(.0010)}$ & $.2210^{(.0024)}$ & $.2568^{(.0156)}$ & $.2828^{(.0037)}$ \\
\hline$b_{i}$ values & $\begin{array}{c}.8365>.5846>.3356>.098 \\
3>0\end{array}$ & $.3502>0$ & $\begin{array}{c}.8310>.5623>.283 \\
4>0\end{array}$ & $.5673>0$ \\
\hline $\mathrm{P}_{f, 0}$ & $3.9280 \mathrm{e}-5^{(2.407 e-6)}$ & $4.0293 \mathrm{e}-5^{(4.0946 e-6)}$ & $1.2030 \mathrm{e}-4^{(1.1022 \mathrm{e}-5)}$ & $1.3187 \mathrm{e}-4^{(5.4984 \mathrm{e}-6)}$ \\
\hline$N_{\text {call }}$ & $5 \mathrm{e} 4$ & $22+11=33$ & $4 \mathrm{e} 4$ & $31+11=42$ \\
\hline
\end{tabular}


Table 4 Results of the sensitivity indices of the second-order cut-HDMR and RS-HDMR component functions for case 2 of the toy test example

\begin{tabular}{|c|c|c|c|c|}
\hline Indices & \multicolumn{2}{|c|}{$S_{\mathrm{Pf}, \mathrm{cut}, i j}$} & \multicolumn{2}{c|}{$S_{\mathrm{Pf}, \mathrm{RS}, i j}$} \\
\hline Methods & LESS-cut-HDMR & AK-LESS-cut-HDMR & GESS-RS-HDMR & AK-GESS-RS-HDMR \\
\hline$\left(\mu_{1}, \mu_{2}\right)$ & $.0002^{(.0000)}$ & $.0002^{(.0000)}$ & $.0007^{(.0000)}$ & $.0008^{(.0000)}$ \\
\hline$\left(\mu_{1}, \sigma_{1}\right)$ & $.0832^{(.0003)}$ & $.0868^{(.0009)}$ & $.1115^{(.0041)}$ & $.0720^{(.0010)}$ \\
\hline$\left(\mu_{1}, \sigma_{2}\right)$ & $.0023^{(.0000)}$ & $.0015^{(.0000)}$ & $.0024^{(.0005)}$ & $.0013^{(.0003)}$ \\
\hline$\left(\mu_{2}, \sigma_{1}\right)$ & $.0043^{(.0000)}$ & $.0031^{(.0000)}$ & $.0024^{(.0001)}$ & $.0028^{(.0001)}$ \\
\hline$\left(\mu_{2}, \sigma_{2}\right)$ & $.0346^{(.0002)}$ & $.0310^{(.0003)}$ & $.0588^{(.0053)}$ & $.0698^{(.0036)}$ \\
\hline$\left(\sigma_{2}, \sigma_{2}\right)$ & $.0034^{(.0000)}$ & $.0037^{(.0001)}$ & $.0070^{(.0062)}$ & $.0027^{(.0007)}$ \\
\hline
\end{tabular}

(a)

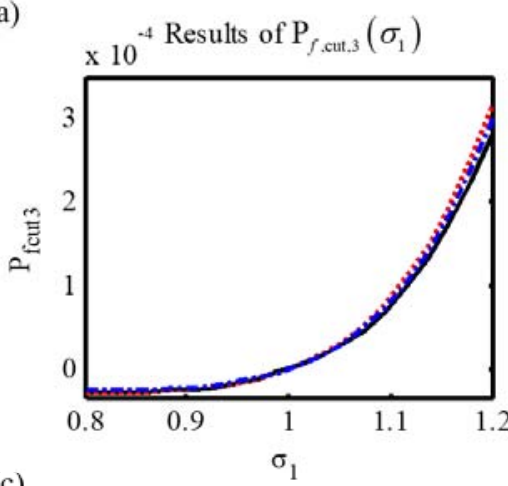

(c)

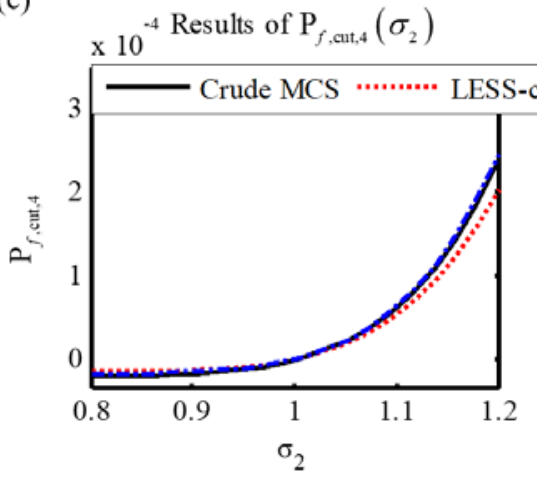

(b)

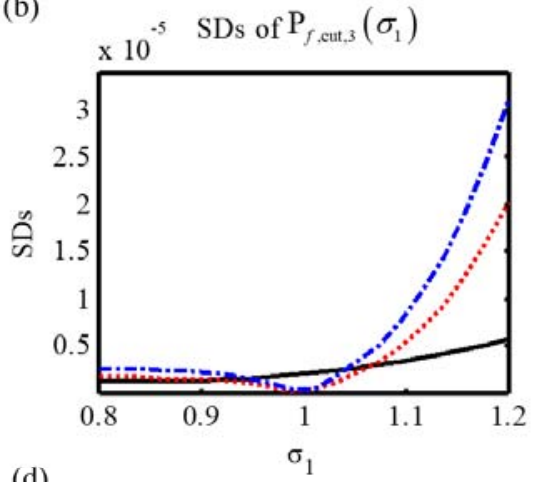

(d)

$\times 10^{-5} \quad$ SDs of $\mathrm{P}_{f, \text { cut }, 4}\left(\sigma_{2}\right)$

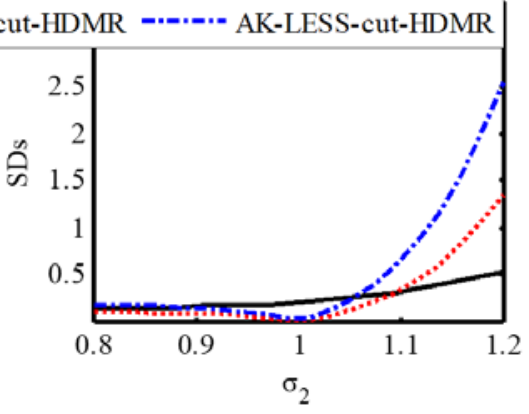

Fig. 5 Results of the first-order influential cut-HDMR component functions for case 2 of the toy test example 
(a)

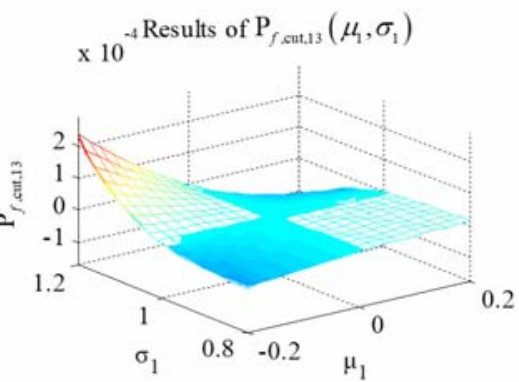

(c)

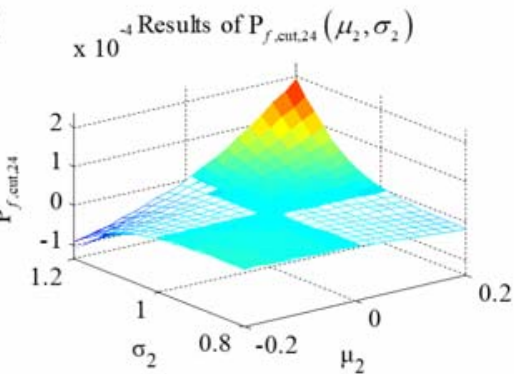

(b)

$\times 10^{-5}$ SDs of $\mathrm{P}_{f, \text { cut.13 }}\left(\mu_{1}, \sigma_{1}\right)$

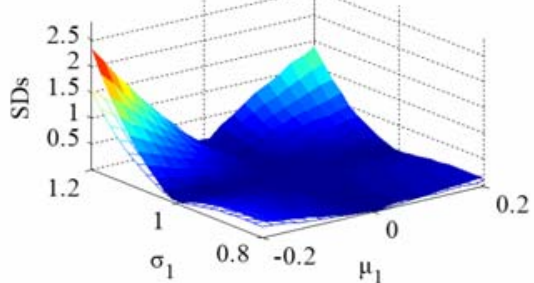

(d) $\quad \times 10^{-5}$ SDs of $\mathrm{P}_{f, \text { sut } 24}\left(\mu_{2}, \sigma_{2}\right)$

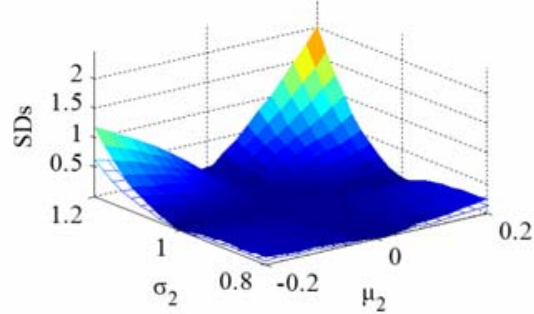

Fig. 6 Results of the second-order influential cut-HDMR component functions for case 2 of the toy test example

(a)

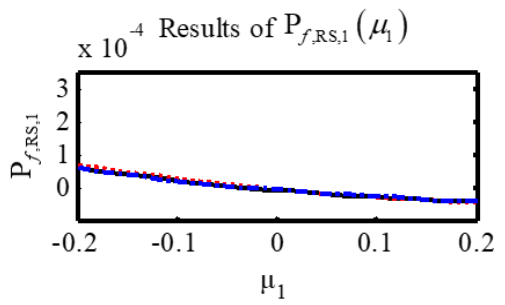

(c)

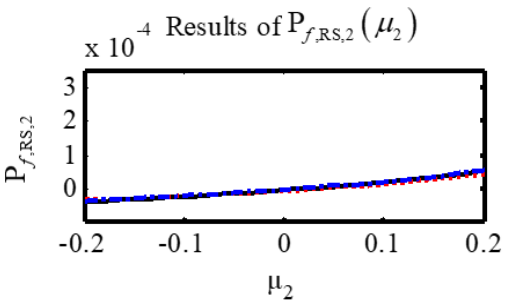

(e)

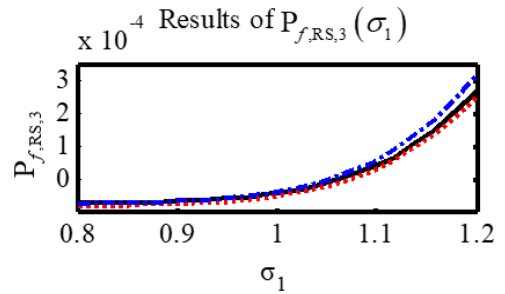

(g)

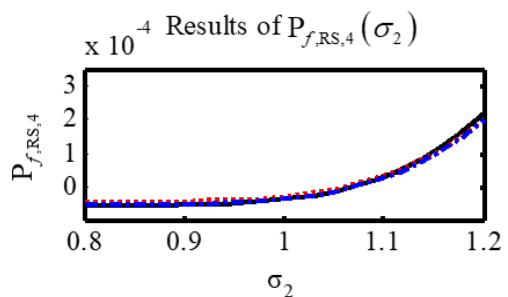

(b)

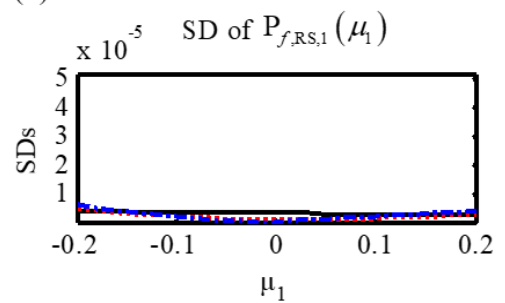

(d)

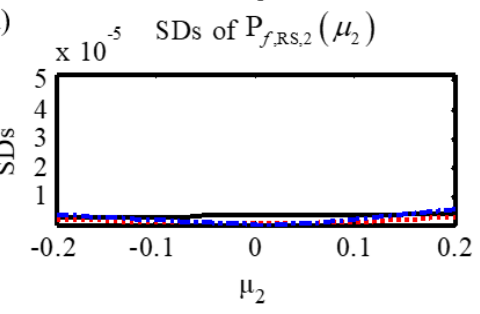

(f) $\times 10^{-5}$ SDs of $\mathrm{P}_{f, \mathrm{RS}, 3}\left(\sigma_{1}\right)$

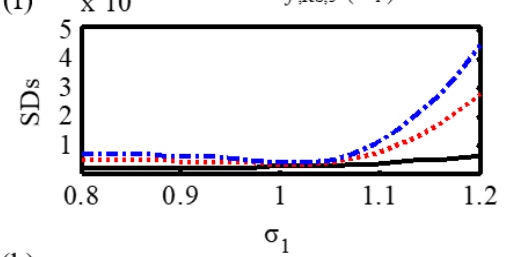

(h)

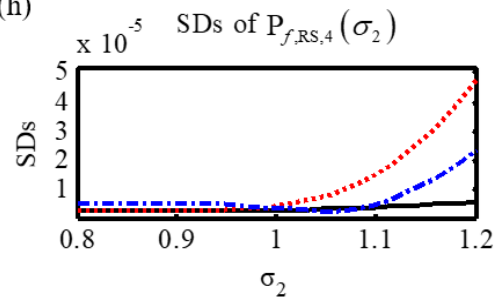

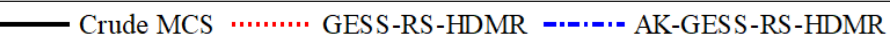

Fig. 7 Results of the first-order RS-HDMR components for case 2 of the toy test example. 
(a)

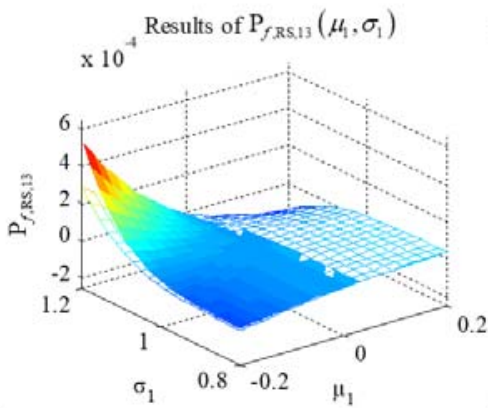

(c)

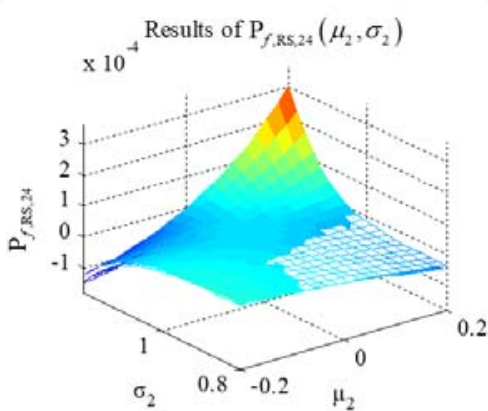

(b)

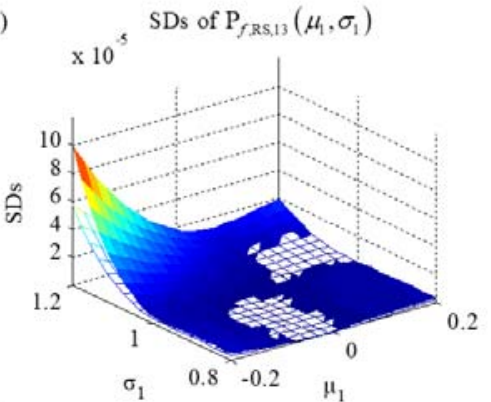

(d)

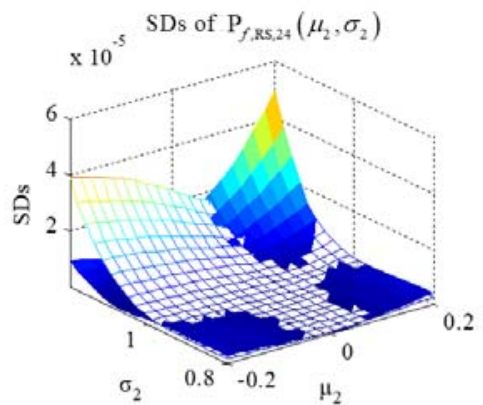

Fig. 8 Results of the second-order influential RS-HDMR component functions estimated for case 2 of the toy test example

\subsection{A planar ten-bar structure}

We still consider the planar ten-bar structure introduced in the companion paper [1]. All the settings for the input variables are the same as that in the mate paper, but the failure threshold is changed to be 0.004 here instead of 0.0035 so as to demonstrate the performances of the methods proposed in this work for rare failure events.

We firstly approximate the failure probability function with the cut-HDMR decomposition. The LESS-cut-HDMR and AK-LESS-cut-HDMR methods are performed with parameter settings shown in the third row of Table 5. The results for the sensitivity indices are reported in the second and third columns of Table 5 and Table 6 , together with the failure thresholds $b_{i}$, the constant components $\mathrm{P}_{f, \text { cut },}$ and the total number of function calls $N_{\text {call }}$. The LESS-cut-HDMR method produces 6 intermediate failure surfaces, while the AK-LESS-HDMR method produce two intermediate failure surfaces, and the total computational costs of these two methods are $3 \mathrm{e} 4$ and 54 respectively. The constant cut-HDMR components computed by both methods match well. It can be seen from the sensitivity indices that only three first-order cut-HDMR components and two second-order component functions are influential, and their results are plotted in Fig. 9 and Fig. 10. It is shown that, all these five component functions are accurately and robustly estimated. Further, the failure probability function is accurately estimated by both methods.

Next we approximate the failure probability function with the RS-HDMR decomposition. The GESSRS-HDMR and AK-GESS-RS-HDMR methods are implemented with the parameters shown in the third row of Table 5, and the sensitivity indices results are displayed in the last two columns of Table 5 and Table 6, from which we can see that only three first-order components and two second-order components are influential, and their results are displayed in Fig. 11 and Fig. 12 respectively. It is shown that all the 
component functions estimated by these two methods match well and their SDs are small, thus the failure probability function is accurately estimated by both methods.

Table 5 Results of first-order sensitivity indices of the ten-bar structure, where the superscripts indicate the results computed with the SDs of the estimates of the corresponding component functions.

\begin{tabular}{|c|c|c|c|c|}
\hline Indices & \multicolumn{2}{|c|}{$S_{\mathrm{Pf}, \mathrm{cut}, i}$} & \multicolumn{2}{c|}{$S_{\mathrm{Pf}, \mathrm{RS}, i}$} \\
\hline Methods & LESS-cut-HDMR & $\begin{array}{c}\text { AK-LESS-cut- } \\
\text { HDMR }\end{array}$ & GESS-RS-HDMR & $\begin{array}{c}\text { AK-GESS-RS- } \\
\text { HDMR }\end{array}$ \\
\hline Settings & $p_{0}=0.1, N=5 \mathrm{e} 3$ & $p_{0}=1 \mathrm{e}-3, N=1 \mathrm{e} 5$ & $p_{0}=0.1, N=5 \mathrm{e} 3$ & $p_{0}=1 \mathrm{e}-3, N=1 \mathrm{e} 5$ \\
\hline$q$ & $.2066^{(.0019)}$ & $.1789^{(.0022)}$ & $.2092^{(.0028)}$ & $.2159^{(.0035)}$ \\
\hline$E$ & $.6143^{(.0057)}$ & $.6549^{(.0080)}$ & $.6041^{(.0071)}$ & $.6215^{(.0094)}$ \\
\hline$P_{1}$ & $.0922^{(.0009)}$ & $.0885^{(.0012)}$ & $.1067^{(.0015)}$ & $.0749^{(.0011)}$ \\
\hline$P_{2}$ & $.0007^{(.0000)}$ & $.0006^{(.0000)}$ & $.0007^{(.0001)}$ & $.0011^{(.0001)}$ \\
\hline$P_{3}$ & $.0000^{(.0000)}$ & $.0000^{(.0000)}$ & $.0001^{(.0000)}$ & $.0001^{(.0000)}$ \\
\hline$b_{i}$ values & $8.025 \mathrm{e}-4,5.490 \mathrm{e}-4$, & $3.570 \mathrm{e}-4,0$ & $8.035 \mathrm{e}-4,5.224 \mathrm{e}-4$, & $3.393 \mathrm{e}-4,0$ \\
& $3.472 \mathrm{e}-4,1.777 \mathrm{e}-4$, & & $3.079 \mathrm{e}-4,1.347 \mathrm{e}-4,0$ & \\
\hline $\mathrm{P}_{f, 0}$ & $1.622 \mathrm{e}-5,0$ & & & $1.165 \mathrm{e}-05^{(1.213 \mathrm{e}-06)}$ \\
\hline$N_{\text {call }}$ & $7.956 \mathrm{e}-6^{(7.570 \mathrm{e}-7)}$ & $7.438 \mathrm{e}-6^{(7.644 \mathrm{e}-7)}$ & $1.338 \mathrm{e}-05^{(1.2333 \mathrm{e}-06)}$ & $34+13=47$ \\
\hline
\end{tabular}

Table 6 Results of the second-order sensitivity indices of the ten-bar structure.

\begin{tabular}{|c|c|c|c|c|}
\hline Indices & \multicolumn{2}{|c|}{$S_{\mathrm{Pf}, \mathrm{cut}, i j}$} & \multicolumn{2}{c|}{$S_{\mathrm{Pf}, \mathrm{RS}, i j}$} \\
\hline Methods & $\begin{array}{c}\text { LESS-cut- } \\
\text { HDMR }\end{array}$ & $\begin{array}{c}\text { AK-LESS-cut- } \\
\text { HDMR }\end{array}$ & $\begin{array}{c}\text { GESS-RS- } \\
\text { HDMR }\end{array}$ & $\begin{array}{c}\text { AK-GESS-RS- } \\
\text { HDMR }\end{array}$ \\
\hline$(q, E)$ & $.0579^{(.0005)}$ & $.0457^{(.0006)}$ & $.0536^{(.0012)}$ & $.0625^{(.0017)}$ \\
\hline$\left(q, P_{1}\right)$ & $.0088^{(.0001)}$ & $.0087^{(.0001)}$ & $.0066^{(.0002)}$ & $.0064^{(.0001)}$ \\
\hline$\left(q, P_{2}\right)$ & $.0000^{(.0000)}$ & $.0000^{(.0000)}$ & $.0001^{(.0000)}$ & $.0000^{(.0000)}$ \\
\hline$\left(q, P_{3}\right)$ & $.0000^{(.0000)}$ & $.0000^{(.0000)}$ & $.0000^{(.0000)}$ & $.0000^{(.0000)}$ \\
\hline$\left(E, P_{1}\right)$ & $.0191^{(.0002)}$ & $.0215^{(.0003)}$ & $.0181^{(.0005)}$ & $.0170^{(.0005)}$ \\
\hline$\left(E, P_{2}\right)$ & $.0001^{(.0000)}$ & $.0001^{(.0000)}$ & $.0003^{(.0001)}$ & $.0001^{(.0000)}$ \\
\hline$\left(E, P_{3}\right)$ & $.0000^{(.0000)}$ & $.0000^{(.0000)}$ & $.0004^{(.0001)}$ & $.0002^{(.00000)}$ \\
\hline$\left(P_{1}, P_{2}\right)$ & $.0000^{(.0000)}$ & $.0000^{(.0000)}$ & $.0000^{(.0000)}$ & $.0001^{(.0000)}$ \\
\hline$\left(P_{1}, P_{3}\right)$ & $.0000^{(.0000)}$ & $.0000^{(.0000)}$ & $.0001^{(.0000)}$ & $.0000^{(.0000)}$ \\
\hline$\left(P_{2}, P_{3}\right)$ & $.0000^{(.0000)}$ & $.0000^{(.0000)}$ & $.0000^{(.0000)}$ & $.0000^{(.0000)}$ \\
\hline
\end{tabular}


(a)

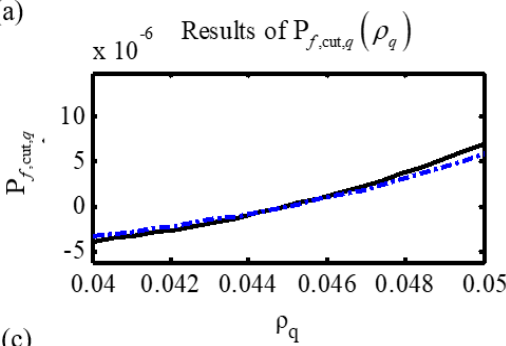

(c)

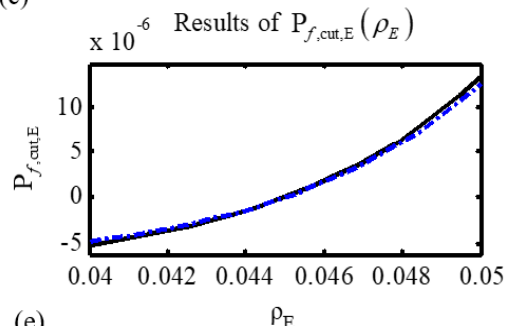

(e)

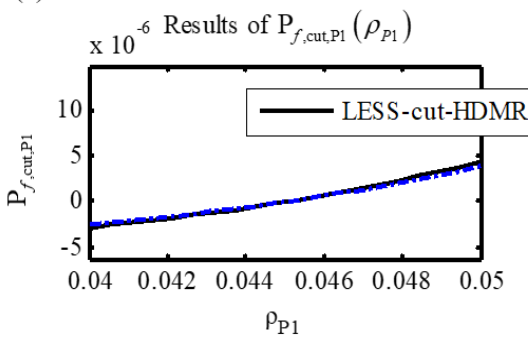

(b)

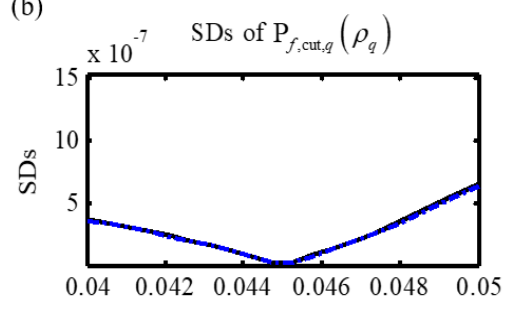

(d)
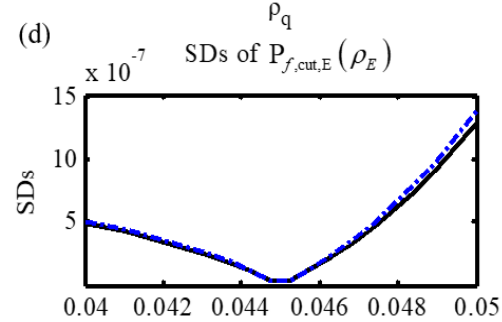

$\rho_{\mathrm{E}}$

(f)

x $10^{-7}$ SDs of $\mathrm{P}_{f, \text { cut } \mathrm{P} 1}\left(\rho_{P 1}\right)$

Fig. 9 Results of the first-order influential cut-HDMR components computed for the ten-bar structure

(a)

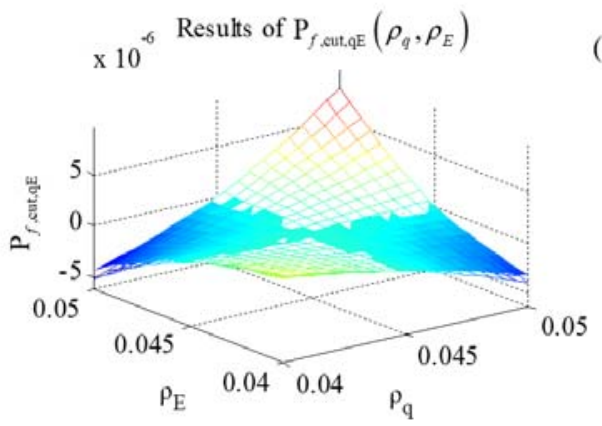

(c)

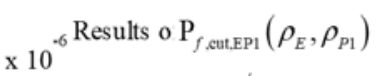

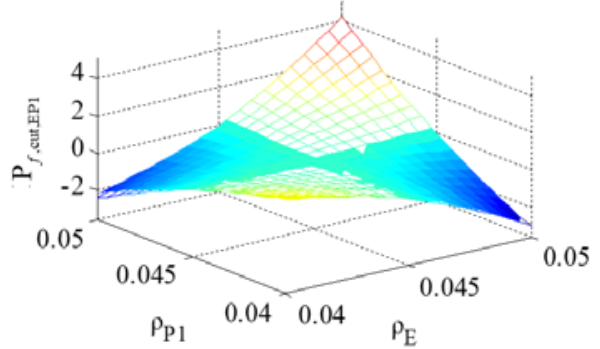

(b)

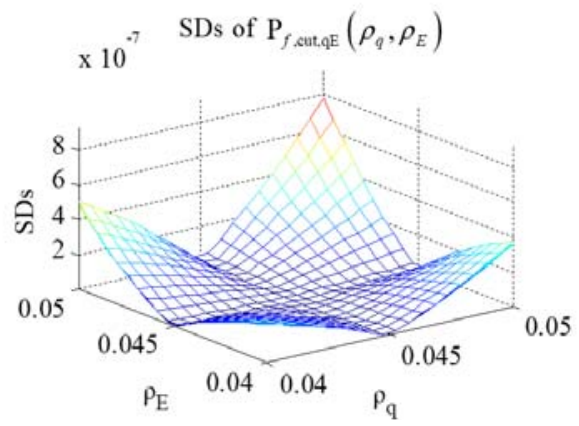

(d)

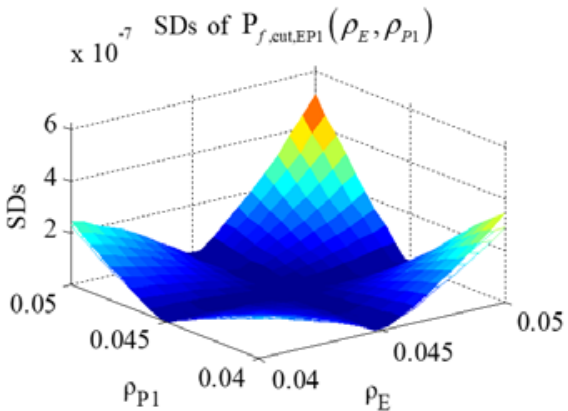

Fig. 10 Results of the second-order influential cut-HDMR component functions estimated for the tenbar structure, where the meshed surfaces are estimated by the LESS-cut-HDMR procedure, and those without mesh are computed by the AK-LESS-cut-HDMR procedure. 
(a)

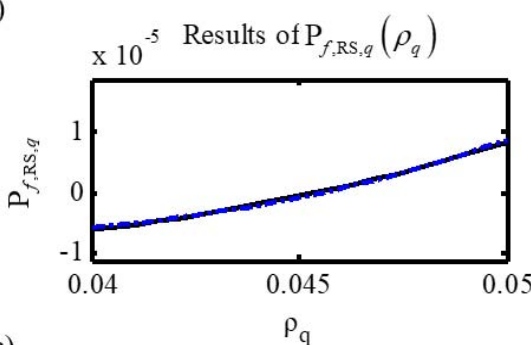

(c)

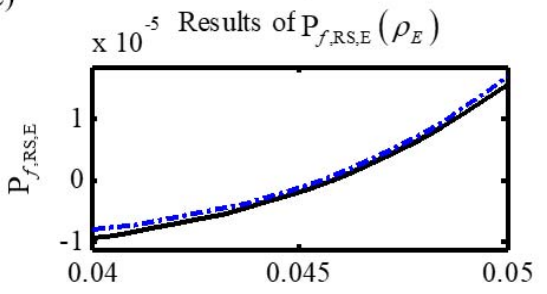

(e)

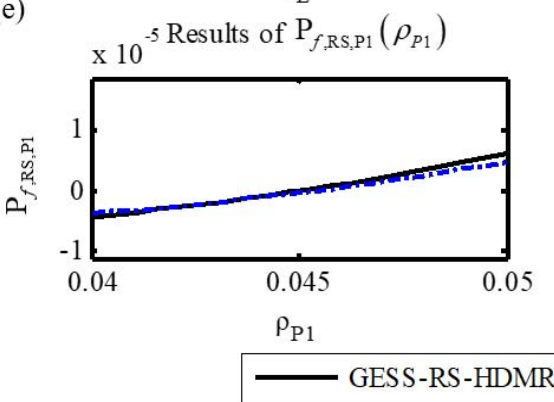

(b)

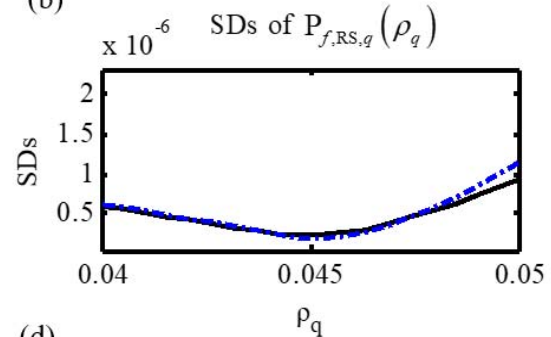

(d)

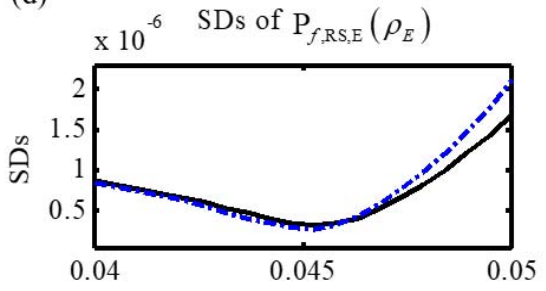

(f) $\quad \times 10^{-6}$ SDs of $\mathrm{P}_{f, \mathrm{RS}, \mathrm{P} 1}\left(\rho_{P 1}\right)$

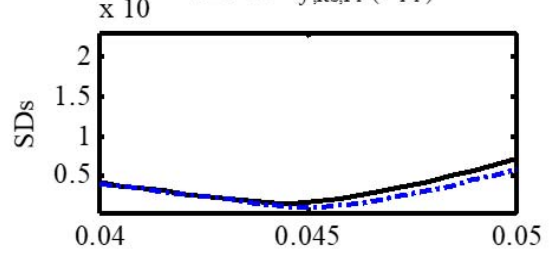

$\rho_{\mathrm{P} 1}$

Fig. 11 Results of the first-order influential RS-HDMR component functions of the ten-bar structure

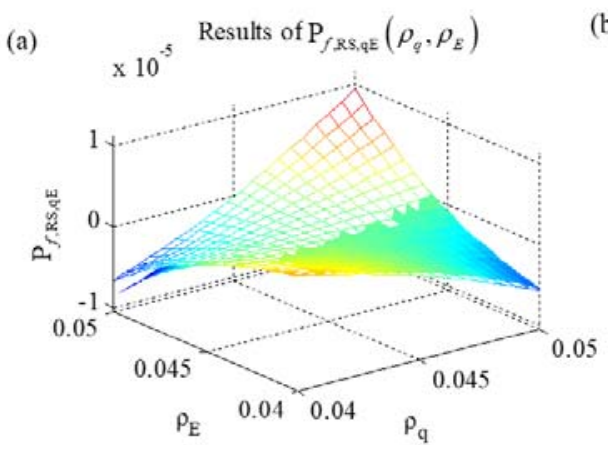

(b)

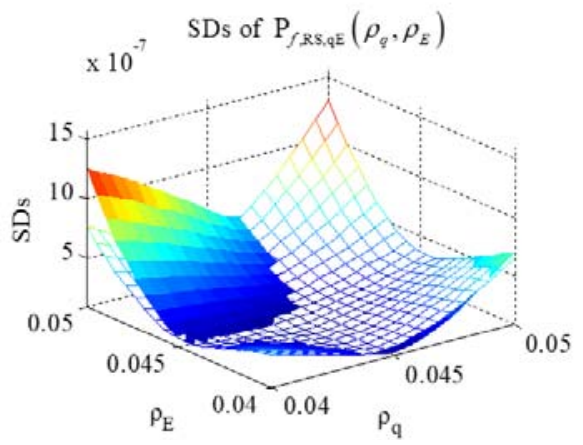

(c)

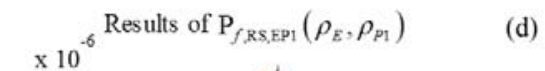

(d)

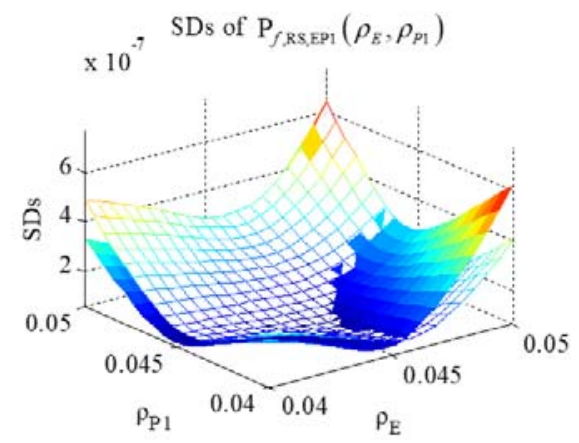

Fig. 12 Results of the second-order influential RS-HDMR component functions of the ten-bar structure. 


\subsection{Application to a two-dimensional wing flutter model}

Consider a two-dimensional wing flutter model adapted from Ref. [41] and shown in Fig. 13, where $G$ refers to the center-of-mass of the wing, $E$ denotes the rigid center, $K_{h}$ and $K_{\alpha}$ are the stiffness of the vertical spring and the torsional spring at the rigid center. Denote the mass of the wing as $m$, and the chord length is $2 b$. The variable $a$ refers to the dimensionless distance between the rigid center and the midpoint of the chord. Let $\mu=m / \pi \rho b^{2}$ indicate the mass ratio, $x_{\alpha}$ denote the dimensionless distance between the points $E$ and $G$. The phugoid mode frequency of the wing is formulated as $\omega_{h}=\sqrt{K_{h} / m}$, and the pitching mode frequency is expressed as $\omega_{\alpha}=\sqrt{K_{\alpha} / m}$. The radius of notation of the wing toward $G$ is denoted as $r_{\alpha}$. Let $h$ and $\alpha$ denote the vertical and rotational displacements respectively. Let $\boldsymbol{q}=(h / b, \alpha)^{T}$ indicate the general displacement, and let $\mathrm{d} \tau=\omega_{\alpha} \mathrm{d} t$ denotes the dimensionless time. Then, the vibration governing equation of the wing is derived as:

$$
\boldsymbol{M} \frac{\mathrm{d}^{2} \boldsymbol{q}}{\mathrm{d} \tau^{2}}+\boldsymbol{K} \boldsymbol{q}=\boldsymbol{F} \boldsymbol{M} \frac{\mathrm{d}^{2} \boldsymbol{q}}{\mathrm{d} \tau^{2}}+\boldsymbol{K} \boldsymbol{q}=\boldsymbol{F}
$$

where $\boldsymbol{M}=\left[\begin{array}{cc}1 & x_{\alpha} \\ x_{\alpha} & r_{\alpha}^{2}\end{array}\right]$ is the mass matrix, $K=\left[\begin{array}{cc}\left(\omega_{h} / \omega_{\alpha}\right) & 0 \\ 0 & r_{\alpha}^{2}\end{array}\right]$ is the stiffness matrix, and $\boldsymbol{F}$ is the generalized aerodynamic force which is expressed as:

$$
\boldsymbol{F}=\frac{v^{2}}{\omega_{\alpha}^{2} b^{2}} \frac{\pi \rho b^{2}}{m} \frac{1}{\pi}\left(\begin{array}{c}
C_{L} \\
2 C_{M_{E}}
\end{array}\right)=\frac{1}{\pi} V_{f}^{* 2}\left(\begin{array}{c}
C_{L} \\
2 C_{M_{E}}
\end{array}\right)
$$

$C_{L}$ and $C_{M_{E}}$ are the aerodynamic force and moment coefficients respectively, and $V_{f}^{*}=V /\left(\omega_{\alpha} b \sqrt{\mu}\right)$ is the dimensionless flutter critical speed. The above flutter model can be solved by the V-g method, and one can refer to subsection 3.7 of Ref. [41] for details. The model output of interest is $V_{f}^{*}$, and commonly, we expect $V_{f}^{*}$ to be large so that the flutter will not happen at normal speed. Based on this fact, we assume that the risk is defined as the probability that $V_{f}^{*}$ does not exceed a threshold (assumed to be 0.4414 in this work), thus the limit state function is defined as $g=V_{f}^{*}-0.4414$. The distribution parameters of six input variables are reported in Table 7. As can be seen, the mean parameter of each input variable is assumed to be constant, but the C.O.V. $\rho_{i}$ of each variable is characterized by interval due to epistemic uncertainty. 


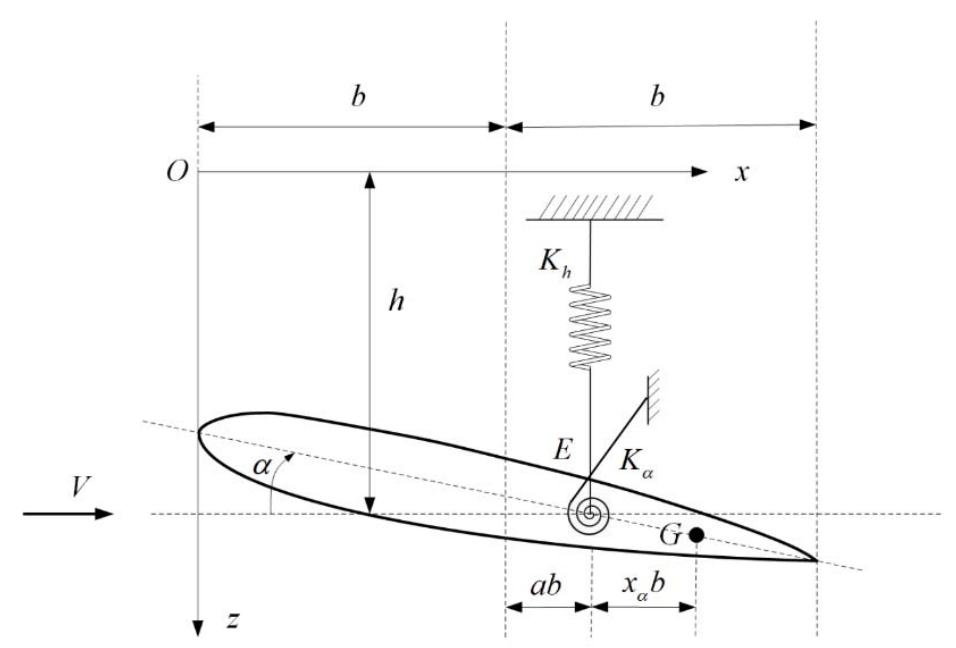

Fig. 13 A two-dimensional wing flutter model

Table 7 Distribution parameters of the input variables of the wing flutter model

\begin{tabular}{|c|c|c|c|c|c|c|}
\hline Input variables & $\mu$ & $r_{\alpha}$ & $\omega_{h}(\mathrm{rad} / \mathrm{s})$ & $a$ & $\omega_{\alpha}(\mathrm{rad} / \mathrm{s})$ & $x_{\alpha}$ \\
\hline Means & 20 & 0.5 & 30 & -0.4 & 50 & 0.2 \\
\hline C.O.V.s & {$[0.035,0.05]$} & {$[0.035,0.05]$} & {$[0.021,0.03]$} & {$[0.021,0.03]$} & {$[0.021,0.03]$} & {$[0.021,0.03]$} \\
\hline
\end{tabular}

We firstly estimate the cut-HDMR component functions and their sensitivity indices for the failure probability function with the AK-LESS-cut-HDMR procedure. The intermediate probability $p_{0}$ is set to be $1 \mathrm{e}-2$, and the sample size $N$ is set to be $3 \mathrm{e} 4$. The method adaptively produces two intermediate failure surfaces with failure thresholds specified as 0.0090 and 0 respectively. The number of function calls for learning the two failure surfaces are 91 and 255 respectively, which are much larger than those of the previous test examples due to the high nonlinearity of the wing flutter model. The constant cut-HDMR component is estimated to be $9.409 \mathrm{e}-4$ with SD being calculated as $5.669 \mathrm{e}-5$. The results for the sensitivity indices of the first-order cut-HDMR component functions are reported in the second row of Table 8 , and the second-order sensitivity indices are all close to zero with very small variations, thus they are not reported due to limited space. As can be seen from Table 8, among the six first-order cut-HDMR component functions, four components (i.e., those of $r_{\alpha}, \omega_{h}, a$ and $\omega_{\alpha}$ ) are influential as the summation of the sensitivity indices of these four components are approximately 0.9812 . Thus the results of these four component functions are displayed in Fig. 14. As can be seen, the results are accurate and robust.

We then estimate the RS-HDMR component functions by the AK-GESS-RS-HDMR procedure. The $p_{0}$ and $N$ are set to be the same as the AK-LESS-cut-HDMR procedure. Similarly, this procedure adaptively produces two intermediate failure surfaces with failure thresholds being calculated as 0.0081 and 0 respectively. The total number of function calls for training these two intermediate failure surfaces are 74 and 305 respectively. The constant RS-HDMR component is estimated to be $1.307 \mathrm{e}-3$ with SD being calculated as 7.766e-5. The sensitivity indices of the first-order component functions are reported in the third row of Table 8. As can be seen, the sensitivity indices of $r_{\alpha}, \omega_{h}, a$ and $\omega_{\alpha}$ add up to 0.9832, thus all the other component functions can be neglected. The results of the four influential component functions are reported in Fig. 15, and are accurate and robust. Thus both the AK-LESS-cut- 
HDMR and AK-GESS-RS-HDMR procedures accurately estimate the failure probability function.

Table 8 The sensitivity indices for the first-order HDMR component functions of the wing flutter models

\begin{tabular}{|c|c|c|c|c|c|c|}
\hline Indices & $\mu$ & $r_{\alpha}$ & $\omega_{h}$ & $a$ & $\omega_{\alpha}$ & $x_{\alpha}$ \\
\hline$S_{\mathrm{Pf}, \mathrm{cut}, i}$ & $.0007^{(.0000)}$ & $.8544^{(.0033)}$ & $.0418^{(.0002)}$ & $.0310^{(.0002)}$ & $.0540^{(.0003)}$ & $.0000^{(.0000)}$ \\
\hline$S_{\mathrm{Pf}, \mathrm{RS}, i}$ & $.0024^{(.0001)}$ & $.8403^{(.0042)}$ & $.0483^{(.0005)}$ & $.0349^{(.0004)}$ & $.0597^{(.0004)}$ & $.0001^{(.0000)}$ \\
\hline
\end{tabular}

(a)

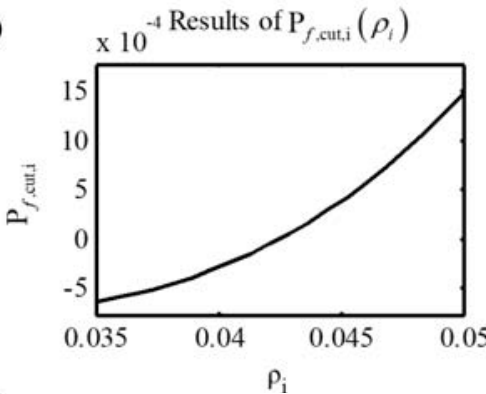

(c)

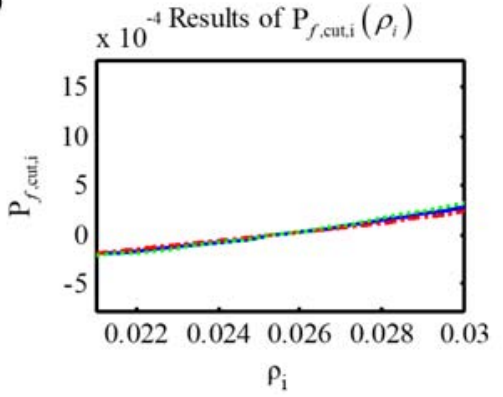

(b) $\times 10^{-5}$ SDs of $\mathrm{P}_{f, \text { cuti. }}\left(\rho_{i}\right)$

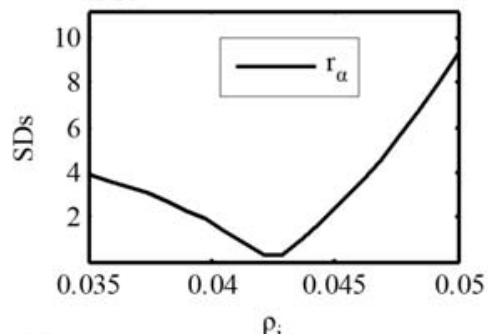

(d)

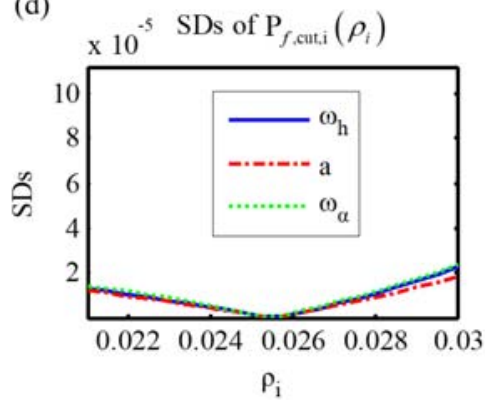

Fig. 14 Results of the influential cut-HDMR component functions of the wing flutter model
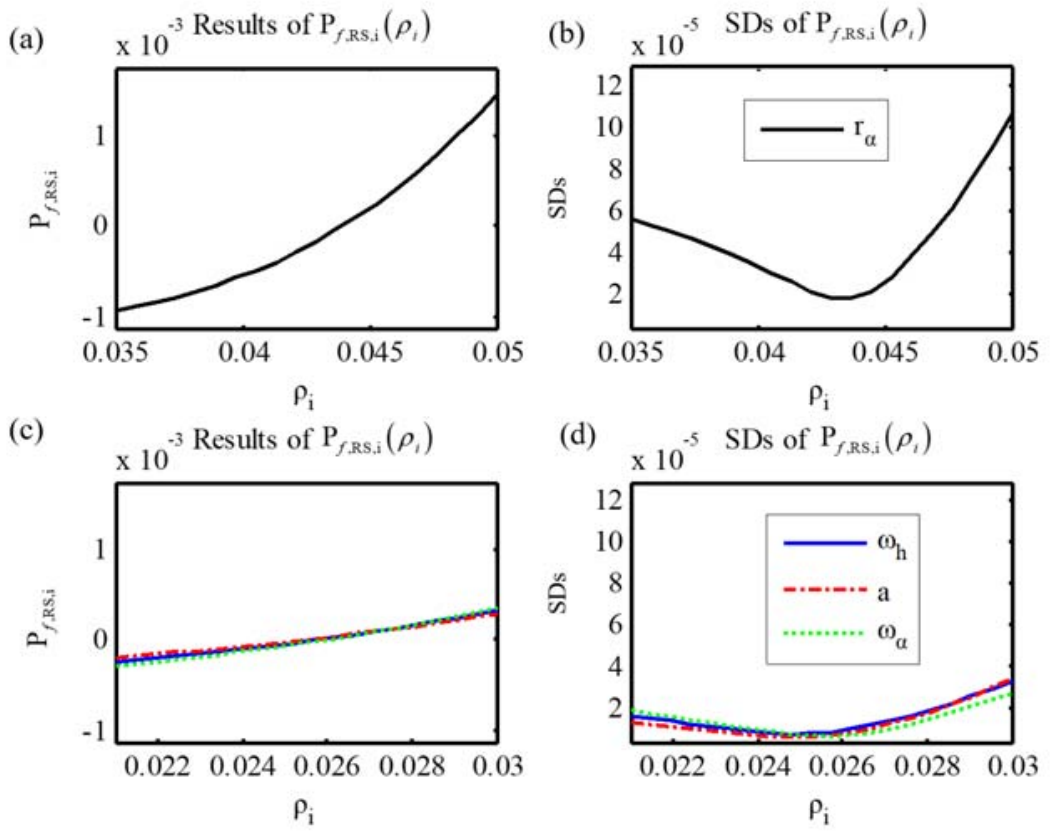

Fig. 15 Results of the influential RS-HDMR component functions of the wing flutter model 


\section{Discussions and Conclusions}

This set of companion paper presents a general methodology framework, called imprecise stochastic simulation, for efficiently propagating the parameterized imprecise probability models. In the companion paper, the framework is developed for response moment function and failure probability function, while in this paper, the estimation of failure probability functions subject to rare failure events is focused, and four algorithms have been developed. Firstly, the LESS-cut-HDMR method for effectively estimating the cut-HDMR components of the failure probability function is developed, and it is further improved to achieve higher efficiency by combining with active learning procedure. These two methods are shown to have desirable local performance. Then, the GESS-RS-HDMR method is developed for estimating the RS-HDMR component functions, and its efficiency is also substantially improved by combining with active learning procedure. These two methods have better global performance. The sensitivity indices are derived for identifying the influential component functions, and measuring the truncation errors of the cut-HDMR and RS-HDMR decompositions. The SDs are derived for quantifying the estimation errors of each component function. The results of the three test examples have shown that the proposed methods are especially effective for structures with rare failure events. Furthermore, the local and global methods can also be combined to improve the estimation accuracy.

The proposed methodology framework can also be extended to be combined with other advanced sampling techniques, such as importance sampling ${ }^{[10][12]}$, line sampling ${ }^{[42]}$ and directional sampling ${ }^{[43]}$, so as to deal with different types of problems, and it can also be used for solving the uncertainty-based design optimization problems ${ }^{[14][27]}$. However, to keep the paper reasonably concise, these extension works are not addressed in this current work. The proposed methodology framework makes the estimation of failure probability bounds and the reliability-based design optimization problem fairly easy to fix. To make the proposed methodology framework more generic, it should also be extended to incorporate any non-parameterized probability models and dependent cases, and this extension will be treated in the future work.

\section{Appendix A: Derivations for LESS-cut-HDMR procedure}

Based on the MC and MCMC sample sets $\mathbf{S}_{q}=\left\{\boldsymbol{x}_{q}^{(k)}: k=1,2, \ldots, N\right\}$, where $q=1,2, \ldots, m$, the LESS estimators of all the cut-HDMR component functions in Eq. (3) are derived as follows. As the intermediate failure surface satisfy $F_{1} \supset F_{2} \supset \ldots \supset F_{m}=F$, the failure probability function can be expressed as:

$$
\mathrm{P}_{f}(\boldsymbol{\theta})=\operatorname{Pr}_{\mathbf{X}}\left(F_{1} \mid \boldsymbol{\theta}\right) \prod_{q=1}^{m} \operatorname{Pr}_{\mathbf{X}}\left(F_{q} \mid F_{q-1}, \boldsymbol{\theta}\right)
$$

where $\operatorname{Pr}_{\mathbf{X}}\left(F_{1} \mid \boldsymbol{\theta}\right)$ indicates the probability measure of the domain $F_{1}$ computed w.r.t. $f_{X}(\boldsymbol{x} \mid \boldsymbol{\theta})$, and $\operatorname{Pr}_{\mathbf{X}}\left(F_{q} \mid F_{q-1}, \theta\right)$ refers to the conditional probability measure of the domain $F_{q}$ computed w.r.t. the conditional joint PDF $f\left(\boldsymbol{x} \mid F_{q-1}, \boldsymbol{\theta}\right)$. These two probability measures can be further derived as ${ }^{[22] \text { : }}$

$$
\operatorname{Pr}_{\mathbf{X}}\left(F_{1} \mid \boldsymbol{\theta}\right)=\int_{\mathrm{R}^{n}} I_{F_{1}}(\boldsymbol{x}) \frac{f_{X}(\boldsymbol{x} \mid \boldsymbol{\theta})}{f_{X}\left(\boldsymbol{x} \mid \boldsymbol{\theta}^{*}\right)} f_{X}\left(\boldsymbol{x} \mid \boldsymbol{\theta}^{*}\right) \mathrm{d} \boldsymbol{x}=\operatorname{Exp}_{\boldsymbol{\theta}^{*}}\left[I_{F_{1}}(\boldsymbol{x}) \frac{f_{X}(\boldsymbol{x} \mid \boldsymbol{\theta})}{f_{X}\left(\boldsymbol{x} \mid \boldsymbol{\theta}^{*}\right)}\right]
$$


and

$$
\begin{aligned}
\operatorname{Pr}_{\mathbf{X}}\left(F_{q} \mid F_{q-1}, \boldsymbol{\theta}\right) & =\int_{\mathbf{R}^{n}} I_{F_{q}}(\boldsymbol{x}) \frac{f_{X}\left(\boldsymbol{x} \mid F_{q-1}, \boldsymbol{\theta}\right)}{f_{\boldsymbol{X}}\left(\boldsymbol{x} \mid F_{q-1}, \boldsymbol{\theta}^{*}\right)} f_{X}\left(\boldsymbol{x} \mid F_{q-1}, \boldsymbol{\theta}^{*}\right) \mathrm{d} \boldsymbol{x} \\
& =\frac{\operatorname{Pr}_{\mathbf{X}}\left(F_{q-1} \mid \boldsymbol{\theta}^{*}\right)}{\operatorname{Pr}_{\mathbf{X}}\left(F_{q-1} \mid \boldsymbol{\theta}\right)} \operatorname{Exp}_{F_{q-1}, \theta^{*}}\left[I_{F_{q}}(\boldsymbol{x}) \frac{f_{X}(\boldsymbol{x} \mid \boldsymbol{\theta})}{f_{X}\left(\boldsymbol{x} \mid \boldsymbol{\theta}^{*}\right)}\right]
\end{aligned}
$$

where $\operatorname{Exp}_{\theta^{*}}[\cdot]$ and $\operatorname{Exp}_{F_{q_{-1}}, \theta^{*}}[\cdot]$ refer the expectation operators w.r.t. joint unconditional PDF $f_{X}\left(\boldsymbol{x} \mid \boldsymbol{\theta}^{*}\right)$ and joint conditional PDF $f_{X}\left(\boldsymbol{x} \mid F_{q-1}, \boldsymbol{\theta}^{*}\right)$ respectively. Further, in Eq. (A3), letting $q=m$ yields,

$$
\begin{aligned}
\mathrm{P}_{f}(\boldsymbol{\theta}) & =\operatorname{Pr}_{\mathbf{X}}\left(F_{m} \mid F_{m-1}, \boldsymbol{\theta}\right) \operatorname{Pr}_{\mathbf{X}}\left(F_{m-1} \mid \boldsymbol{\theta}\right)=\operatorname{Pr}_{\mathbf{X}}\left(F_{m-1} \mid \boldsymbol{\theta}^{*}\right) \operatorname{Exp}_{F_{m-1}, \boldsymbol{\theta}^{*}}\left[I_{F_{m}}(\boldsymbol{x}) \frac{f_{X}(\boldsymbol{x} \mid \boldsymbol{\theta})}{f_{X}\left(\boldsymbol{x} \mid \boldsymbol{\theta}^{*}\right)}\right] \\
& =\operatorname{Pr}_{\mathbf{X}}\left(F_{1} \mid \boldsymbol{\theta}^{*}\right) \prod_{q=2}^{m-1} \operatorname{Pr}_{\mathbf{X}}\left(F_{q} \mid F_{q-1}, \boldsymbol{\theta}^{*}\right) \operatorname{Exp}_{F_{m-1}, \theta^{*}}\left[I_{F_{m}}(\boldsymbol{x}) \frac{f_{X}(\boldsymbol{x} \mid \boldsymbol{\theta})}{f_{\boldsymbol{X}}\left(\boldsymbol{x} \mid \boldsymbol{\theta}^{*}\right)}\right]
\end{aligned}
$$

Based on the above derivation, the cut-HDMR component functions of $\mathrm{P}_{f}(\boldsymbol{\theta})$ can be derived as

$$
\left\{\begin{array}{l}
\mathrm{P}_{f, \text { cut }, 0}=\operatorname{Pr}_{\mathbf{X}}\left(F_{1} \mid \boldsymbol{\theta}^{*}\right) \prod_{q=2}^{m} \operatorname{Pr}_{\mathbf{X}}\left(F_{q} \mid F_{q-1}, \boldsymbol{\theta}^{*}\right) \\
\mathrm{P}_{f, \text { cut }, i}\left(\theta_{i}\right)=\operatorname{Pr}_{\mathbf{X}}\left(F_{1} \mid \boldsymbol{\theta}^{*}\right) \prod_{q=2}^{m-1} \operatorname{Pr}_{\mathbf{X}}\left(F_{q} \mid F_{q-1}, \boldsymbol{\theta}^{*}\right) \operatorname{Exp}_{F_{m-1}, \boldsymbol{\theta}^{*}}\left[I_{F}(\boldsymbol{x}) r_{\text {cut }, i}\left(\boldsymbol{x} \mid \theta_{i}, \boldsymbol{\theta}^{*}\right)\right] \\
\mathrm{P}_{f, \text { cut }, j}\left(\boldsymbol{\theta}_{i j}\right)=\operatorname{Pr}_{\mathbf{X}}\left(F_{1} \mid \boldsymbol{\theta}^{*}\right) \prod_{q=2}^{m-1} \operatorname{Pr}_{\mathbf{X}}\left(F_{q} \mid F_{q-1}, \boldsymbol{\theta}^{*}\right) \operatorname{Exp}_{F_{m-1}, \theta^{*}}\left[I_{F}(\boldsymbol{x}) r_{\text {cut }, j}\left(\boldsymbol{x} \mid \theta_{i j}, \boldsymbol{\theta}^{*}\right)\right]
\end{array}\right.
$$

, and further their LESS estimators can be derived as:

$$
\left\{\begin{array}{l}
\hat{\mathrm{P}}_{f, \text { cut }, 0}=p_{0}^{m-1}\left[\frac{1}{N} \sum_{k=1}^{N} I_{F_{m}}\left(\boldsymbol{x}_{m}^{(k)}\right)\right] \\
\hat{\mathrm{P}}_{f, \text { cut }, i}\left(\theta_{i}\right)=p_{0}^{m-1}\left[\frac{1}{N} \sum_{k=1}^{N} I_{F_{m}}\left(\boldsymbol{x}_{m}^{(k)}\right) r_{\text {cut }, i}\left(\boldsymbol{x}_{m}^{(k)} \mid \theta_{i}, \boldsymbol{\theta}^{*}\right)\right] \\
\hat{\mathrm{P}}_{f, \text { cut }, j}\left(\boldsymbol{\theta}_{i j}\right)=p_{0}^{m-1}\left[\frac{1}{N} \sum_{k=1}^{N} I_{F_{m}}\left(\boldsymbol{x}_{m}^{(k)}\right) r_{\text {cut }, i j}\left(\boldsymbol{x}_{m}^{(k)} \mid \boldsymbol{\theta}_{i j}, \boldsymbol{\theta}^{*}\right)\right]
\end{array}\right.
$$

\section{Appendix B: Derivations for GESS-RS-HDMR procedure}

Based on the joint MC and MCMC sample sets $\mathbf{S}_{q}=\left\{\left(\boldsymbol{x}_{q}^{(k)}, \boldsymbol{\theta}_{q}^{(k)}\right): k=1,2, \ldots, N\right\}(q=1,2, \ldots, m)$, the GESS estimators for all the RS-HDMR component functions are as follows. Let $\boldsymbol{\theta}^{\prime}=\left(\theta_{1}^{\prime}, \theta_{2}^{\prime}, \ldots, \theta_{m}^{\prime}\right)^{T}$ indicate the random replication of $\boldsymbol{\theta}$, which means that $\boldsymbol{\theta}^{\prime}$ and $\boldsymbol{\theta}$ are independent, and have the same distribution. Then, the conditional probability $\operatorname{Pr}_{\mathbf{X}, \boldsymbol{\Theta}}\left(F_{q} \mid F_{q-1}\right)$ computed with respect to $\boldsymbol{x}$ and $\boldsymbol{\theta}^{\prime}$ can be derived as:

$$
\operatorname{Pr}_{\mathbf{X}, \boldsymbol{\Theta}}\left(F_{q} \mid F_{q-1}\right)=\int_{\mathbf{R}^{n+d}} I_{F_{q}}(\boldsymbol{x}) f_{X, \boldsymbol{\Theta}}\left(\boldsymbol{x}, \boldsymbol{\theta}^{\prime} \mid F_{q-1}\right) \mathrm{d} \boldsymbol{x} \mathrm{d} \boldsymbol{\theta}^{\prime}=\int_{\mathbf{R}^{n+d}} I_{F_{q}}(\boldsymbol{x}) \frac{f_{X, \boldsymbol{\Theta}}\left(\boldsymbol{x}, \boldsymbol{\theta}^{\prime}\right)}{\mathrm{P}_{X, \boldsymbol{\Theta}}\left(F_{q-1}\right)} \mathrm{d} \boldsymbol{x} \mathrm{d} \boldsymbol{\theta}^{\prime}
$$


Based on Eq. (A7), the constant component term $\mathrm{P}_{f, \mathrm{RS}, 0}$ can be derived as:

$$
\begin{aligned}
\mathrm{P}_{f, \mathrm{RS}, 0} & =\int_{\mathbf{R}^{n+d}} I_{F_{m}}(\boldsymbol{x}) f\left(\boldsymbol{x}, \boldsymbol{\theta}^{\prime}\right) \mathrm{d} \boldsymbol{x} \mathrm{d} \boldsymbol{\theta}^{\prime}=\operatorname{Pr}_{\mathbf{X}, \boldsymbol{\Theta}}\left(F_{m} \mid F_{m-1}\right) \operatorname{Pr}_{\mathbf{X}, \boldsymbol{\Theta}}\left(F_{m-1}\right) \\
& =\operatorname{Pr}_{\mathbf{X}, \boldsymbol{\Theta}}\left(F_{1}\right) \prod_{q=2}^{m} \operatorname{Pr}_{\mathbf{X}, \boldsymbol{\Theta}}\left(F_{q} \mid F_{q-1}\right) \\
& \hat{=} p_{0}^{m-1}\left[\frac{1}{N} \sum_{k=1}^{N} I_{F_{m}}\left(\boldsymbol{x}_{m}^{(k)}\right)\right]
\end{aligned}
$$

For the first-order RS-HDMR component function, the conditional probability $\operatorname{Pr}_{\mathbf{x}, \boldsymbol{\Theta}_{-i}}\left(F_{q} \mid F_{q-1}, \theta_{i}\right)$ computed w.r.t. $\boldsymbol{x}$ and $\boldsymbol{\theta}_{-i}^{\prime}$ can be derived as:

$$
\begin{aligned}
\operatorname{Pr}_{\mathbf{X}, \boldsymbol{\Theta}_{-i}}\left(F_{q} \mid F_{q-1}, \theta_{i}\right) & =\int_{\mathbf{R}^{n+d-1}} I_{F_{q}}(\boldsymbol{x}) f_{X, \boldsymbol{\Theta}_{-i}}\left(\boldsymbol{x}, \boldsymbol{\theta}_{-i} \mid F_{q-1}, \theta_{i}\right) \mathrm{d} \boldsymbol{x} \mathrm{d} \boldsymbol{\theta}_{-i} \\
& =\frac{1}{\operatorname{Pr}_{\mathbf{X}, \boldsymbol{\Theta}}\left(F_{q-1}\right)} \int_{\mathbf{R}^{n+d-1}} I_{F_{q}}(\boldsymbol{x}) f_{\boldsymbol{X}, \boldsymbol{\Theta}_{-i}}\left(\boldsymbol{x}, \boldsymbol{\theta}_{-i} \mid \theta_{i}\right) \mathrm{d} \boldsymbol{x} \mathrm{d} \boldsymbol{\theta}_{-i}
\end{aligned}
$$

Then, letting $q=m$ in Eq. (A9) yields,

$$
\begin{aligned}
\mathrm{P}_{f, \mathrm{RS}, i}\left(\theta_{i}\right) & =\operatorname{Pr}_{\mathbf{X}, \boldsymbol{\Theta}}\left(F_{m-1}\right) \operatorname{Pr}_{\mathbf{X}, \boldsymbol{\Theta}_{-i}}\left(F_{m} \mid F_{m-1}, \theta_{i}\right)-\mathrm{P}_{f, \mathrm{RS}, 0} \\
& =\operatorname{Pr}_{\mathbf{X}, \boldsymbol{\Theta}}\left(F_{1}\right)\left[\prod_{q=2}^{m-1} \operatorname{Pr}_{\mathbf{X}, \boldsymbol{\Theta}}\left(F_{q} \mid F_{q-1}\right)\right] \int_{\mathbf{R}^{n+d-1}} I_{F_{m}}(\boldsymbol{x}) f_{\boldsymbol{X}, \boldsymbol{\Theta}_{-i}}\left(\boldsymbol{x}, \boldsymbol{\theta}_{-i}^{\prime} \mid F_{m-1}, \theta_{i}\right) \mathrm{d} \boldsymbol{x} \mathrm{d} \boldsymbol{\theta}_{-i}^{\prime}-\mathrm{P}_{f, \mathrm{RS}, 0} \\
& =p_{0}^{m-1} \int_{\mathbf{R}^{n+d-1}} I_{F_{m}}(\boldsymbol{x}) \frac{f_{\boldsymbol{X}, \boldsymbol{\Theta}_{-i}}\left(\boldsymbol{x}, \boldsymbol{\theta}_{-i}^{\prime} \mid F_{m-1}, \theta_{i}\right)}{f_{\boldsymbol{X}, \boldsymbol{\Theta}_{-i}}\left(\boldsymbol{x}, \boldsymbol{\theta}_{-i}^{\prime} \mid F_{m-1}, \theta_{i}^{\prime}\right)} f_{\boldsymbol{X}, \boldsymbol{\theta}_{-i}}\left(\boldsymbol{x}, \boldsymbol{\theta}_{-i}^{\prime} \mid F_{m-1}, \theta_{i}^{\prime}\right) \mathrm{d} \boldsymbol{x} \mathrm{d} \boldsymbol{\theta}_{-i}^{\prime}-\mathrm{P}_{f, \mathrm{RS}, 0} \\
& =p_{0}^{m-1} \int_{\mathbf{R}^{n+d}} I_{F_{m}}(\boldsymbol{x})\left[\frac{f_{\boldsymbol{X}, \boldsymbol{\Theta}_{-i}}\left(\boldsymbol{x}, \boldsymbol{\theta}_{-i}^{\prime} \mid \theta_{i}\right)}{f_{\boldsymbol{X}, \boldsymbol{\theta}_{-i}}\left(\boldsymbol{x}, \boldsymbol{\theta}_{-i}^{\prime} \mid \theta_{i}^{\prime}\right)}-1\right] f_{\boldsymbol{X}, \boldsymbol{\Theta}_{-i}}\left(\boldsymbol{x}, \boldsymbol{\theta}^{\prime} \mid F_{m-1}\right) \mathrm{d} \boldsymbol{x} \mathrm{d} \boldsymbol{\theta}^{\prime} \\
& \hat{=} p_{0}^{m-1}\left[\frac{1}{N} \sum_{k=1}^{N} I_{F_{m}}\left(\boldsymbol{x}_{m}^{(k)}\right) r_{\mathrm{RS}, i}\left(\boldsymbol{x}_{m}^{(k)} \mid \theta_{i}, \boldsymbol{\theta}_{m}^{(k)}\right)\right]
\end{aligned}
$$

Similarly, the GESS estimators for the second-order RS-HDMR component functions can be derived as:

$$
\mathrm{P}_{f, \mathrm{RS}, i j}\left(\boldsymbol{\theta}_{i j}\right) \hat{=} p_{0}^{m-1}\left[\frac{1}{N} \sum_{k=1}^{N} I_{F_{m}}\left(\boldsymbol{x}_{m}^{(k)}\right) r_{\mathrm{RS}, i j}\left(\boldsymbol{x}_{m}^{(k)}, \boldsymbol{\theta}_{i j}, \boldsymbol{\theta}_{m}^{(k)}\right)\right]
$$

The GESS estimators for higher order RS-HDMR component functions can be similarly derived, and don't repeat them.

\section{Acknowledgements}

This work is supported by the Natural Science Basic Research Plan in Shaanxi Province of China (Grant No. 2017JQ1007) and the Aerospace Science and Technology Foundation of China. The first and third authors are both supported by the Alexander von Humboldt Foundation of Germany. The first author is also supported by the Top International University Visiting Program for Outstanding Young scholars of Northwestern Polytechnical University. The second author is supported by the program of China Scholarships Council (CSC). The authors are thankful for all these grants. The authors would also like to thank Prof. Hongshuang Li for providing the Matlab code of subset simulation, Dr. Xiaojing Wu for providing the wing flutter model and the two anonymous reviewers for helpful comments.

\section{Reference}

[1] P.F. Wei, J.W. Song, S.F. Bi, M. Broggi, M. Beer, Non-intrusive stochastic analysis with 
parameterized imprecise probability models: I. performance estimation, Mech. Syst. Signal Process. in press, doi:10.1016/j.ymssp.2019.01.058

[2] M. Beer, S. Ferson, V. Kreinovich, Imprecise probabilities in engineering analyses, Mech. Syst. Signal Process. 37 (2013) 4-29

[3] K. Sentz, S. Ferson, Combination of evidence in Dempster-Shafer theory, Livermore, California: Sandia National Laboratories, 2002

[4] S. Sun, G. Fu, S. Djordjević, S.T. Khu, Separating aleatory and epistemic uncertainties: Probabilistic sewer flooding evaluation using probability box, J. Hydrol. 420 (2012) 360-372.

[5] M. Stein, M. Beer, V. Kreinovich, Bayesian approach for inconsistent information, Inform. Sciences 245(2013) 96-111

[6] S. Sankararaman, S. Mahadevan, Likelihood-based representation of epistemic uncertainty due to sparse point data and/or interval data, Reliab. Eng. Syst. Safe. 96 (2011) 814-824.

[7] J. Mi, Y. F. Li, Y. J. Yang, W. Peng, H. Z. Huang, Reliability assessment of complex electromechanical systems under epistemic uncertainty. Reliab. Eng. Syst. Safe., 152(2016), 1-15.

[8] A.M. Hasofer, NC. Lind, An exact and invariant first order reliability format, J. Eng. Mech. 100(1974) 111-121.

[9] I. Lee, Y. Noh, D. Yoo, A novel second-order reliability method (SORM) using noncentral or generalized chi-squared distribution, J. Mech. Design 134 (2012) 100912

[10] S.K. Au, J.L. Beck, A new adaptive important sampling scheme, Struct. Saf. 21(1999) 135-158.

[11] S.K. Au, J.L. Beck, Estimation of small failure probabilities in high dimensions by subset simulation, Probabilist. Eng. Mech. 16(2001) 263-277.

[12] W. Yun, Z. Lu, X. Jiang, A modified importance sampling method for structural reliability and its global reliability sensitivity analysis. Struct Multidiscip Optim 57(2018) 1625-1641.

[13] B. Echard, N. Gayton, M. Lemaire, AK-MCS: an active learning reliability method combining kriging and Monte Carlo simulation, Struct. Saf. 33(2011) 145-154.

[14] L. Li, H. Wan, W. Gao, F. Tong, H. Li,. Reliability based multidisciplinary design optimization of cooling turbine blade considering uncertainty data statistics. Struct Multidiscip Optim 59(2018) 115.

[15] P. Wei, F. Liu, C. Tang, Reliability and reliability-based importance analysis of structural systems using multiple response Gaussian process model, Reliab. Eng. Syst. Safe. 175 (2018) 183-195.

[16] V. Dubourg, B. Sudret, F. Deheeger, Metamodel-based importance sampling for structural reliability analysis, Probabilist. Eng. Mech. 33(2013) 47-57.

[17] M. Faes, M. Broggi, E. Patelli, Y. Govers, J. Mottershead, M. Beer, D. Moens, A multivariate interval approach for inverse uncertainty quantification with limited experimental data, Mech. Syst. Signal Process 118(2019), 534-548.

[18] A. Jalal-Kamali, V. Kreinovich, Estimating correlation under interval uncertainty, Mech. Syst. Signal Process 37(2013) 43-53.

[19] X. Y. Long, C. Jiang, X. Han, J. C. Tang, F. J. Guan, An enhanced subinterval analysis method for uncertain structural problems, Appl. Math. Model. 54(2018) 580-593.

[20] H. Zhang, H. Dai, M. Beer, W. Wang, Structural reliability analysis on the basis of small samples: 
an interval quasi-Monte Carlo method, Mech. Syst. Signal Process. 37 (2013) 137-151

[21] S.K. Au, Reliability-based design sensitivity by efficient simulation, Comput. Struct. 83 (2005)1048-61

[22] X.K. Yuan, Local estimation of failure probability function by weighted approach, Probabilist. Eng. Mech. 34 (2013) 1-11

[23] Z. Zhang, C. Jiang, G.G. Wang, X. Han, First and second order approximate reliability analysis methods using evidence theory, Reliab. Eng. Syst. Safe. 137 (2015) 40-49

[24] H.B. Liu, C. Jiang, X.Y. Jia, X.Y. Long, Z. Zhang, F. J. Guan, A new uncertainty propagation method for problems with parameterized probability-boxes, Reliab. Eng. Syst. Safe. 172(2018) 64-73

[25] X. Yang, Y. Liu, P. Ma, Structural reliability analysis under evidence theory using the active learning kriging model, Eng. Optimiz. 49 (2017) 1922-1938.

[26] R. Schöbi, B. Sudret, Structural reliability analysis for p-boxes using multi-level metamodels, Probabilist. Eng. Mech. 48(2017) 27-38.

[27] P. Wei, Z. Lu, J. Song, Extended Monte Carlo simulation for parametric global sensitivity analysis and optimization, AIAA J. 52 (2014) 867-878.

[28] P.F. Wei, Z. Z. Lu, J. W. Song, Variable importance analysis: a comprehensive review. Reliab. Eng. Syst. Safe. 142(2015) 399-342

[29] W. Yun, Z. Lu, X. Jiang, An efficient sampling approach for variance-based sensitivity analysis based on the law of total variance in the successive intervals without overlapping. Mech. Syst. Signal Process, 106(2018) 495-510.

[30] D.A. Alvarez, F. Uribe, J.E. Hurtado, Estimation of the lower and upper bounds on the probability of failure using subset simulation and random set theory, Mech. Syst. Signal Process 100 (2018) 782-801

[31] P. Wei, F. Liu, Z. Lu, Z. Wang, A probabilistic procedure for quantifying the relative importance of model inputs characterized by second-order probability models, Int. J. Approx. Reason. 98 (2018) 78-95

[32] B.J. Bichon, M.S. Eldred, L.P. Swiler, S. Mahadevan, J.M. McFarland, Efficient global reliability analysis for nonlinear implicit performance functions, AIAA J. 46 (2008) 2459-2468

[33] W.K. Hastings, Monte Carlo sampling methods using Markov chains and their applications, Biometrika 57 (1970) 97-109

[34] M. Rosenblatt, Remarks on a Multivariate Transformation, Ann. Math. Stat. 23(1952) 470-472

[35] R. Lebrun, A. Dutfoy, An innovating analysis of the Nataf transformation from the copula viewpoint, Probabilist. Eng. Mech. 24 (2009) 312-320.

[36] A.M. Santoso, K.K. Phoon, ST. Quek, Modified Metropolis-Hastings algorithm with reduced chain correlation for efficient Subset Simulation, Probabilist. Eng. Mech. 26(2011) 331-341

[37] F. Miao, M. Ghosn, Modified Subset Simulation method for reliability analysis of structural systems, Struct. Saf. 33(2011) 251-260.

[38] I. Papaioannou, W. Betz, K. Zwirglmaier, D. Straub, MCMC algorithms for Subset Simulation, Probabilist. Eng. Mech. 44(2015) 89-103

[39] S.K. Au, E. Patelli, Rare event simulation in finite-infinite dimensional space, Reliab. Eng. Syst. 
Safe. 148(2016) 67-77

[40] P.F. Wei, C.H. Tang, Structural reliability and reliability sensitivity analysis of extremely rare failure events by combining sampling and surrogate model methods, P. I. Mech. O- J. Ris. revision submitted

[41] Z.Y. Ye, W.W. Zhang, A.M. Shi, Fundamentals of Fluid-Structure Coupling and Its Application, Harbin, China: Harbin Institute of Technology Press, 2010

[42] G.I. Schuëller, H.J. Pradlwarter, P.S. Koutsourelakis, A critical appraisal of reliability estimation procedures for high dimensions, Probabilist. Eng. Mech. 19 (2004) 463-473

[43] N. Jinsuo, B.R. Ellingwood, Directional methods for structural reliability analysis, Struct. Saf. 22(2000) 233-249 